Karl-Franzens Universität Graz

Technische Universität Graz

Medizinische Universität Graz

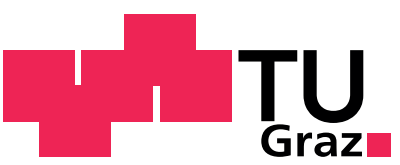

\title{
A discrepancy-based parameter adaptation and stopping rule for minimization algorithms aiming at Tikhonov-type regularization
}

\author{
K. Bredies \\ M. Zhariy
}


SFB sponsors:

- Austrian Science Fund (FWF)

- University of Graz

- Graz University of Technology

- Medical University of Graz

- Government of Styria

- City of Graz

Das Land

Steiermark

Stadt $\mathbf{G} \mathbf{R}$ A Z $\mathbf{Z}$ Wissenschaft 


\title{
A discrepancy-based parameter adaptation and stopping rule for minimization algorithms aiming at Tikhonov-type regularization
}

\author{
Kristian Bredies \\ Institute for Mathematics and Scientific Computing, University of Graz, Heinrichstraße 36, \\ A-8010 Graz, Austria \\ E-mail: kristian.bredies@uni-graz.at
}

\section{Mariya Zhariy}

Software Competence Center Hagenberg, Softwarepark 21, A-4232 Hagenberg, Austria

E-mail: mariya.zhariy@scch.at

12 July 2012

\begin{abstract}
We present a discrepancy-based parameter choice and stopping rule for iterative algorithms performing approximate Tikhonov-functional minimization which adapts the regularization parameter value during the optimization procedure. The suggested parameter choice and stopping rule can be applied to a wide class of penalty terms and iterative algorithms which aim at Tikhonov regularization with a fixed parameter value. It leads, in particular, to computable guaranteed estimates for the regularized exact discrepancy in terms of numerical approximations. Based on these estimates, convergence to a solution is shown.

As an example, the developed theory and the algorithm is applied to the case of sparse regularization. We prove order optimal convergence rates in case of sparse regularization, i.e., weighted $\ell^{p}$ norms, which turn out to be the same as for the a-priori parameter choice rule already obtained in the literature as well as for Morozov's principle applied to exact regularized solutions. Finally, numerical results for two different minimization techniques, iterative soft thresholding (ISTA) and monotone fast iterative soft thresholding (MFISTA), are presented, confirming, in particular, the results from the theory.
\end{abstract}

AMS classification scheme numbers: 65J10, 65J20, 49M29.

Keywords: Tikhonov-type regularization, iterative regularization, duality based methods, parameter adaptation, stopping rules, a-posteriori parameter choice. 


\section{Introduction}

Consider a linear, bounded, ill-posed operator $A: X \rightarrow Y$ mapping between some Hilbert spaces $X$ and $Y$. Ill-posed means that the solution of the equation

$$
A x=y
$$

does not continuously depend on the data $y$. Typically, only some perturbed version $y^{\delta}$ with $\left\|y-y^{\delta}\right\|_{Y} \leq \delta$ is known which makes the direct inversion impossible. An established technique for solving the inverse problem nevertheless is Tikhonov regularization. It yields approximations of the sought $x$ by finding a minimizer of the Tikhonov functional $T_{\alpha}^{\delta}$ given by

$$
T_{\alpha}^{\delta}(x)=\frac{1}{2}\left\|A x-y^{\delta}\right\|_{Y}^{2}+\alpha \phi(x),
$$

where $\phi$ is a penalty functional and $\alpha$ a regularization parameter. Such a minimization is usually stable with respect to the perturbed data $y^{\delta}$. However, the problem of choosing the regularization parameter $\alpha$ appropriately remains. While there are many strategies such as a-priori parameter choice rules, it is useful, from the practical point of view, to adapt the parameter according to the outcome of the regularization method, i.e., to choose it a-posteriori. Morozov's discrepancy principle is, for instance, such a strategy. It bases on adjusting the discrepancy $\left\|A x_{\alpha}^{\delta}-y^{\delta}\right\|_{Y}$ where $x_{\alpha}^{\delta}$ is a minimizer of $T_{\alpha}^{\delta}$ with respect to the noise level $\delta$. If one wants to apply this principle in practice, one has, however, to know the exact minimizers $x_{\alpha}^{\delta}$. Typically, numerical algorithms for minimizing Tikhonov functionals are of iterative nature and only yield approximations to a minimizer for a fixed $\alpha$. Consequently, only an approximation of the discrepancy is available provided that sufficiently many iterations of the algorithm are carried out. Additionally, to find the parameter a-posteriori, one usually has to perform the minimization procedure several times which can be quite time-consuming.

In this paper we propose a general framework to overcome these practical problems. More precisely, we define a discrepancy based parameter adaptation and stopping rule which acts on top of a given iterative minimization procedure for Tikhonov-type functionals. It is applicable to a wide class of optimization algorithms as well as to general penalty terms. It gives in particular computable estimates for the accuracy of the discrepancy of the exact minimizer in terms of the iterates. It will be shown that, as an iterative procedure, it is itself regularizing meaning that it converges to a solution of the original inverse problem for a vanishing noise level. This framework can in particular be applied to inverse problems with weighted $\ell^{p}$ regularization which also covers sparsity constraints. In this particular case, the proposed procedure also yields order optimal convergence rates under appropriate source conditions and assumptions on the forward operator $A$. It can directly be combined with commonly used minimization algorithms such as the iterative soft-thresholding algorithm (ISTA) [1] or the monotone fast iterative soft-thresholding (MFISTA) [2]. 
Before going into details, let us shortly review some recent results about a-posteriori parameter choice rules and iterative as well as sparse regularization. In the last years, several results have been obtained for the Morozov discrepancy principle in case of general Tikhonovtype regularization, cf. [3] for linear inverse problems and [4] for nonlinear inverse problems. The mentioned results are mainly of theoretical nature, since they assume the knowledge of exact minimizers of the Tikhonov functionals. From the practical side where one has only available inexact solutions from numerical algorithms, one can mention [5], where Morozov's discrepancy principle was applied as a stopping rule to the Landweber iteration. In order to reduce the computational effort and to obtain sparse approximations, this discrepancy principle has moreover been used to define a hard shrinkage parameter in each step of the Landweber iteration as well as a stopping criterion, see [6, 7]. The thresholding operation introduces a perturbation in the iteration procedure and may be interpreted as an error source in the problem representation. In case of modeling errors, a modified discrepancy principle, depending on the modelling error, has been developed in [8,9]. Some further ideas on implementable approaches for the sparse solution of inverse problems have been presented in [10-13]. Finally, convergence and especially convergence rates for the Tikhonov-type regularization in Banach spaces have been considered by several authors, e.g. [1, 14-21]. For the particular case of the weighted $\ell_{p}$-regularization, an a-priori parameter choice rule $\alpha=\alpha(\delta)$ has been proposed in [1].

The article is organized as follows: In Section 2 we summarize the basic assumptions on the problem and the regularizing Tikhonov functional. Furthermore, we assume existence of an iterative minimization algorithm

$$
x_{k+1}=\mathcal{P}^{\alpha, \delta}\left(x_{k}\right), \quad x_{0} \in \operatorname{dom}\left(T_{\alpha}^{\delta}\right), \quad k \geq 0
$$

for the Tikhonov functional $T_{\alpha}^{\delta}$.

The main requirement on the minimization algorithm is that it converges in terms of the functional values, i.e., the functional remainder $r\left(x_{k}\right)=T_{\alpha}^{\delta}\left(x_{k}\right)-T_{\alpha}^{\delta}\left(x_{\alpha}^{\delta}\right)$ has to vanish as $k \rightarrow \infty$. Unfortunately, the functional remainder depends on the unknown minimizer $x_{\alpha}^{\delta}$. Therefore, we introduce another quantity, the primal-dual gap $D\left(x_{k}\right)$, which is a computable upper bound on $r\left(x_{k}\right)$, and show that it can replace the functional remainder in the discrepancy estimates.

Recall that, as we in general only reach at the exact minimizers in the limit, the calculation of the exact discrepancy $\left\|A x_{\alpha}^{\delta}-y^{\delta}\right\|$, needed to find the regularization parameter $\alpha$ in the classical approach, is not possible. Therefore, we have to estimate the exact discrepancy by some computable quantities.

In Section 3 we derive estimates for the the exact discrepancy from below and from above

$$
L B \leq\left\|A x_{\alpha}^{\delta}-y^{\delta}\right\| \leq U B
$$

where the lower bound $L B$ and the upper bound $U B$ only depend on the inexact discrepancy $\left\|A x_{k}-y^{\delta}\right\|$ and the primal-dual gap $D\left(x_{k}\right)$. With the convergence of the primal-dual gap and 
the fact that $r\left(x_{k}\right) \rightarrow 0$ the obtained bounds converge towards the exact discrepancy as $k \rightarrow \infty$. It means that we can precisely determine when the exact discrepancy is approximated with any prescribed accuracy.

In Section 4, the announced discrepancy principle is formulated. It aims at the adaptation of the regularization parameter $\alpha$ during the minimizing iteration and yields a stopping criterion at the same time. Since the exact discrepancy is not available, we replace it by the estimates from Section 3.

\section{Parameter Adaptation}

If the exact discrepancy $\left\|A x_{\alpha}^{\delta}-y^{\delta}\right\|$ satisfies

$$
\left\|A x_{\alpha}^{\delta}-y^{\delta}\right\|>\tau \delta
$$

the classical approach suggests to reduce $\alpha$ by multiplying it with some factor $\kappa<1$. In our case, we check if the lower bound satisfies

$$
L B\left(x_{k}\right)>\tau \delta .
$$

As in this case $\left\|A x_{\alpha}^{\delta}-y^{\delta}\right\|>\tau \delta$ holds, we update the parameter value. As the lower bound approaches the exact discrepancy as $k \rightarrow \infty$, we always adapt the parameter after a finite number of iteration steps.

\section{Stopping Criterion}

The classical discrepancy principle suggests to stop the iteration once

$$
\left\|A x_{\alpha}^{\delta}-y^{\delta}\right\| \leq \tau \delta
$$

for some fixed $\tau>1$. We replace this criterion by

$$
U B<\tau(1+\sigma) \delta
$$

where $\sigma>0$ is some small number. We introduce $\sigma$ to deal with the situation $\left\|A x_{\alpha}^{\delta}-y^{\delta}\right\|=\tau \delta$, since in this case we will possibly neither obtain $U B<\tau \delta$ nor $L B>\tau \delta$ after a finite number of iteration steps. As in the parameter adaptation case, the stopping criterion will be reached after a finite number of iteration steps, once the exact discrepancy, corresponding to the effective value of $\alpha$, is small enough.

Section 4 is concluded by a regularization result, i.e. it is shown that the resulting approximations converge towards the generalized solution as $\delta \rightarrow 0$.

In Section 5, we derive order optimal convergence rates for exact Morozov discrepancy principle in case of the weighted $\ell_{p}$ regularization in the same framework as in [1]. In particular, we consider a source condition, which treats the smoothness of the solution independently from the properties of the operator. This kind of separate source condition is typical e.g. for regularization in Hilbert scales [22].

In Section 6 we illustrate the proposed regularization method by means of a numerical example which confirms its practical applicability, in particular, the results from the previous sections. The paper is concluded in Section 7 with some remarks. 


\section{Basic Assumptions}

In the following, we present the basic assumptions for our Tikhonov-minimization problem and the minimization algorithm for a fixed regularization parameter $\alpha>0$. The truncated iteration procedure will turn out to be essentially independent from the minimization method. We are posing the following assumptions on the problem:

Assumption 2.1. The Tikhonov functional

$$
T_{\alpha}^{\delta}=F^{\delta}+\alpha \phi \quad, \quad F^{\delta}(x)=\frac{1}{2}\left\|A x-y^{\delta}\right\|^{2},
$$

satisfies the following properties:

(i) the functional $\phi: \ell_{2} \rightarrow[0, \infty]$ is proper, convex, lower semi-continuous and coercive,

(ii) it holds that $\phi(x)=0$ if and only if $x=0$,

(iii) the forward operator $A$ is in $\mathcal{L}\left(\ell_{2}, Y\right)$ with $Y$ being a Hilbert space,

(iv) there exists exact data $y^{\dagger}=$ Ax for some $x \in \ell_{2}$ with $\phi(x)<\infty$ and for each $\delta>0$, perturbed data $y^{\delta} \in Y$ with $\left\|y^{\dagger}-y^{\delta}\right\| \leq \delta$.

Note that these assumptions immediately imply, for each $\delta>0$ and $\alpha>0$, the existence of minimizers in $\ell_{2}$, we will denote such a minimizer by $x_{\alpha}^{\delta}$. Also, the set of minimum- $\phi$ solutions

$$
\mathcal{S}^{\dagger}=\left\{x^{\dagger} \in \ell_{2}: A x^{\dagger}=y^{\dagger}, \phi\left(x^{\dagger}\right)=\min _{A x=y^{\dagger}} \phi(x)\right\}
$$

is non-empty. We will typically denote by $x^{\dagger}$ an element of $\mathcal{S}^{\dagger}$. Finally, they make sure that the classical Morozov discrepancy principle is regularizing (confer Section 4).

In addition to the Tikhonov functional embedded into the framework of convex optimization, we assume that we have an algorithm available which reduces the functional values in a controlled way as well as a so-called primal-dual gap which will be used to control the regularization parameter in the truncated iteration procedure.

Assumption 2.2. For each $\alpha>0, \delta>0$, there is given a minimization algorithm $\mathcal{P}^{\alpha, \delta}=$ $\left\{\mathcal{P}_{1}^{\alpha, \delta}, \mathcal{P}_{2}^{\alpha, \delta}, \ldots\right\}$ for $T_{\alpha}^{\delta}$ which produces, for each $x_{0} \in \operatorname{dom}\left(T_{\alpha}^{\delta}\right)$, a sequence $x_{k}=\mathcal{P}_{k}^{\alpha, \delta}\left(x_{0}\right)$ such that

(i) the functional distance

$$
r(x)=T_{\alpha}^{\delta}(x)-\min _{\tilde{x} \in \ell_{2}} T_{\alpha}^{\delta}(\tilde{x}),
$$

abbreviated $r_{k}=r\left(x_{k}\right)$, satisfies

$$
r_{k}<\infty \quad \text { for all } k \geq 1 \quad \text { and } \quad \lim _{k \rightarrow \infty} r_{k}=0 \text {, }
$$

(ii) there exists a $\tilde{\psi}_{\alpha}: \ell_{2} \rightarrow[0, \infty]$ proper, convex, lower semi-continuous which may depend on $x_{0}$, such that 
(a) we have $\tilde{\psi}_{\alpha}(0)=0$ and $\tilde{\psi}_{\alpha} \geq \alpha \phi$,

(b) each minimizer $x_{\alpha}^{\delta}$ of $T_{\alpha}^{\delta}$ also minimizes $\tilde{T}_{\alpha}^{\delta}$ according to

$$
\tilde{T}_{\alpha}^{\delta}=F^{\delta}+\tilde{\psi}_{\alpha},
$$

(c) the functional distance for $\tilde{T}_{\alpha}^{\delta}$, i.e., $\tilde{r}=\tilde{T}_{\alpha}^{\delta}-\min \tilde{T}_{\alpha}^{\delta}$, satisfies, with $\tilde{r}_{k}=\tilde{r}\left(x_{k}\right)$ the identity $\tilde{r}_{k}=r_{k}$ for all $k \geq 1$,

(d) the Fenchel dual

$$
\tilde{\psi}_{\alpha}^{*}(z)=\sup _{x \in \ell_{2}}\langle z, x\rangle-\tilde{\psi}_{\alpha}(x)
$$

is Lipschitz continuous on a set containing $\left\{-A^{*}\left(A x_{k}-y^{\delta}\right)\right\}_{k} \cup\left\{-A^{*}\left(A x_{\alpha}^{\delta}-y^{\delta}\right)\right\}$.

Definition 2.3. In the situation of Assumption 2.2, the functional $D: \ell_{2} \rightarrow[0, \infty]$ defined by

$$
D(x)=\left\langle A^{*}\left(A x-y^{\delta}\right), x\right\rangle+\tilde{\psi}_{\alpha}(x)+\tilde{\psi}_{\alpha}^{*}\left(-A^{*}\left(A x-y^{\delta}\right)\right),
$$

is the primal-dual gap associated to $\tilde{T}_{\alpha}^{\delta}$. Furthermore, define $D_{k}=D\left(x_{k}\right)$ for $k \geq 1$ the primaldual gap in the iterates.

We are also interested in convergence speed and therefore occasionally assume rates in terms of functional descent.

Assumption 2.4. The algorithm from Assumption 2.2 obeys the estimate

$$
r_{k} \leq C k^{-\rho}
$$

for all $k \geq 1$ and some $C>0$ as well as $\rho>0$ independent from $k$.

Note that Assumption 2.1 covers all linear inverse problems with proper, convex and lower semi-continuous regularization term. The descent estimate in Assumption 2.2 is, as it will be discussed later in this section, satisfied for many minimization algorithms. The condition on the Fenchel dual and primal-dual gap is just ensuring that we can estimate $r_{k}$ in terms of the computable value $D_{k}$ which also tends to zero (as we will see subsequently). We assume that the evaluation of $\tilde{\psi}_{\alpha}$ as well as $\tilde{\psi}_{\alpha}^{*}$ is computationally accessible.

Let us, at this point, take a closer look at the functional $\tilde{\psi}_{\alpha}$ and the associated primal-dual gap in view of Assumption 2.2. We begin with an easy sufficient condition for the items (ii)a, (ii)b and (ii)c, i.e., $\tilde{T}_{\alpha}^{\delta}$ including the minimizers of $T_{\alpha}^{\delta}$ and $\tilde{r}_{k}=r_{k}$ for all $k \geq 1$ : Let $\tilde{\psi}_{\alpha}$ proper, convex and lower semi-continuous such that $\tilde{\psi}_{\alpha}(0)=0$,

$$
\tilde{\psi}_{\alpha} \geq \alpha \phi \quad \text { and } \quad \tilde{\psi}_{\alpha}(x)=\alpha \phi(x) \quad \text { for each } x\left\{\begin{array}{l}
\text { minimizer of } T_{\alpha}^{\delta} \text { and } \\
x=x_{k}, k \geq 1 \text { iterate. }
\end{array}\right.
$$

It is immediate that in this case, $\tilde{T}_{\alpha}^{\delta} \geq T_{\alpha}^{\delta}$ and $\min _{x \in \ell_{2}} \tilde{T}_{\alpha}^{\delta}=\min _{x \in \ell_{2}} T_{\alpha}^{\delta}$ as well as $\tilde{r}_{k}=r_{k}$ for all $k \geq 1$.

Next, we mention a sufficient condition for item (ii)d in Assumption 2.2, i.e., for $\tilde{\psi}_{\alpha}^{*}$ being Lipschitz continuous on bounded sets. 
Proposition 2.5. Let $\left.\left.\tilde{\psi}_{\alpha}: \ell_{2} \rightarrow\right]-\infty, \infty\right]$ be proper, convex, lower semi-continuous and strongly coercive, i.e.,

$$
\|x\| \rightarrow \infty \quad \Rightarrow \quad \frac{\tilde{\psi}_{\alpha}(x)}{\|x\|} \rightarrow \infty,
$$

then $\tilde{\psi}_{\alpha}^{*}$ is Lipschitz continuous on bounded sets, in particular, on each set containing $\left\{-A^{*}\left(A x_{k}-y^{\delta}\right)\right\}_{k} \cup\left\{-A^{*}\left(A x_{\alpha}^{\delta}-y^{\delta}\right)\right\}$.

Proof. We show that $\tilde{\psi}_{\alpha}^{*}(z)<\infty$ for each $z \in \ell_{2}$. This implies, since it is a convex function, the desired Lipschitz continuity on bounded sets [23]. Therefore, let $z \in \ell_{2}$ be given. Observe that by strong coercivity, one can find an $L>0$ such that $\|z\| \leq \tilde{\psi}_{\alpha}(x) /\|x\|$ for each $\|x\| \geq L$. Hence,

$$
\sup _{\|x\| \geq L}\langle z, x\rangle-\tilde{\psi}_{\alpha}(x) \leq \sup _{\|x\| \geq L}\|x\|\left(\|z\|-\frac{\tilde{\psi}_{\alpha}(x)}{\|x\|}\right) \leq 0 .
$$

On the other hand, we have that the functional $G$ defined by

$$
G(x)=\tilde{\psi}_{\alpha}(x)-\langle z, x\rangle+I_{\{\|\cdot\| \leq L\}}(x)
$$

is convex, lower semi-continuous and coercive and hence admits a minimum $M$. Consequently,

$$
\sup _{\|x\| \leq L}\langle z, x\rangle-\tilde{\psi}_{\alpha}(x) \leq-M,
$$

and, together with the above, it follows that $\tilde{\psi}_{\alpha}(z)<\infty$.

Finally, since $\lim _{k \rightarrow \infty} r_{k}=0, F^{\delta}\left(x_{k}\right)=\frac{1}{2}\left\|A x_{k}-y^{\delta}\right\|^{2}$ is bounded and consequently, $\left\{-A^{*}\left(A x_{k}-\right.\right.$ $\left.\left.y^{\delta}\right)\right\}_{k} \cup\left\{-A^{*}\left(A x_{k}-y^{\delta}\right)\right\}$ is a bounded set.

Finally, we observe that under Assumption 2.2, the primal-dual gap according to (3) always estimates the functional distance $\tilde{r}$. As a preparation, recall the definition of the subdifferential as well as the optimality conditions for minimizers of $T_{\alpha}^{\delta}$.

Definition 2.6. Let $X$ be a real Hilbert space and $G: X \rightarrow]-\infty, \infty]$ be proper, convex and lower semi-continuous. Then, for $z, x \in X$ we say $z$ is in the subgradient of $G$ at $x$, denoted $z \in \partial G(x)$, if and only if for each $y \in X$, the subgradient inequality

$$
G(x)+\langle z, y-x\rangle \leq G(y)
$$

is satisfied. The set-valued mapping $\partial G$ is called the subdifferential of $G$.

This notion provides a convenient way of characterizing minimizers, in particular, we know that:

Lemma 2.7. An $x \in \ell_{2}$ is a minimizer for $T_{\alpha}^{\delta}$ if and only if

$$
-A^{*}\left(A x-y^{\delta}\right) \in \alpha \partial \phi(x) .
$$


Proof. This is a consequence of standard subdifferential calculus: $x$ is a minimizer if and only if $0 \in \partial(F+\alpha \phi)(x)=\partial F(x)+\alpha \partial \phi(x)$ where $\partial F(x)=\left\{F^{\prime}(x)\right\}=\left\{A^{*}\left(A x-y^{\delta}\right)\right\}$.

For more details on convex analysis and subdifferential calculus, we refer the reader to, e.g., $[23,24]$.

Proposition 2.8. Let $\left.\left.\tilde{\psi}_{\alpha}: \ell_{2} \rightarrow\right]-\infty, \infty\right]$ be proper, convex and lower semi-continuous and consider $\tilde{T}_{\alpha}^{\delta}$ according to (2). If there exists a minimizer of $\tilde{T}_{\alpha}^{\delta}$, then we have, with $D$ according to (3), that

$$
0 \leq \tilde{r} \leq D
$$

Proof. Denote by $x_{*}$ a minimizer of $\tilde{T}_{\alpha}^{\delta}$. The subgradient inequality for $F$ gives, rearranged

$$
F^{\delta}(x)-F^{\delta}\left(x_{*}\right) \leq\left\langle A^{*}\left(A x-y^{\delta}\right), x-x_{*}\right\rangle .
$$

Plugging this into $\tilde{T}_{\alpha}^{\delta}$ and using the definition of the Fenchel dual yields

$$
\begin{aligned}
\tilde{r}(x)= & \tilde{T}_{\alpha}^{\delta}(x)-\tilde{T}_{\alpha}^{\delta}\left(x_{*}\right) \\
\leq & \left\langle A^{*}\left(A x-y^{\delta}\right), x\right\rangle+\tilde{\psi}_{\alpha}(x)+\left(\left\langle-A^{*}\left(A x-y^{\delta}\right), x_{*}\right\rangle-\tilde{\psi}_{\alpha}\left(x_{*}\right)\right) \\
\leq & \left\langle A^{*}\left(A x-y^{\delta}\right), x\right\rangle+\tilde{\psi}_{\alpha}(x) \\
& +\sup _{\tilde{x} \in \ell_{2}}\left(\left\langle-A^{*}\left(A x-y^{\delta}\right), \tilde{x}\right\rangle-\tilde{\psi}_{\alpha}(\tilde{x})\right)=D(x) .
\end{aligned}
$$

Now, one important application we are in particular interested in is the situation of inverse problems with sparsity constraints. The following example shows that Assumptions 2.1 and 2.2 are indeed satisfied for the widely-used iterative-thresholding algorithm.

Example 2.9. Let $A \in \mathcal{L}\left(\ell_{2}, Y\right)$ for some Hilbert space $Y, 1 \leq p \leq 2$ and $\phi(x)=\|x\|_{p}^{p}$. Note that $\phi$ is proper, convex, lower semi-continuous and coercive. Therefore, for each $y^{\dagger} \in \operatorname{rg} A$, there exists a minimum-norm solution $x^{\dagger}$. Furthermore, each Tikhonov functional

$$
T_{\alpha}^{\delta}(x)=\frac{1}{2}\left\|A x-y^{\delta}\right\|^{2}+\alpha\|x\|_{p}^{p},
$$

is coercive, hence meaning that Assumption 2.1 is satisfied, if we choose noisy data $y^{\delta}$ for each $\delta>0$ accordingly.

Moreover, many algorithms have been proposed for its numerical minimization [1, 25-29], here, we mention the popular iterative soft-thresholding algorithm (ISTA) by [1]:

$$
\left\{\begin{aligned}
x_{0} & \in \ell_{p} \\
x_{k+1} & =\mathbf{S}_{\alpha p, p}\left[x_{k}-\tau A^{*}\left(A x_{k}-y^{\delta}\right)\right]
\end{aligned}\right.
$$

where $0<\tau<2 /\|A\|^{2}$. Moreover, $\mathbf{S}_{\alpha, p}$ denotes the componentwise application of the $p$-softthresholding function $S_{\alpha, p}$ which, in turn, amounts to

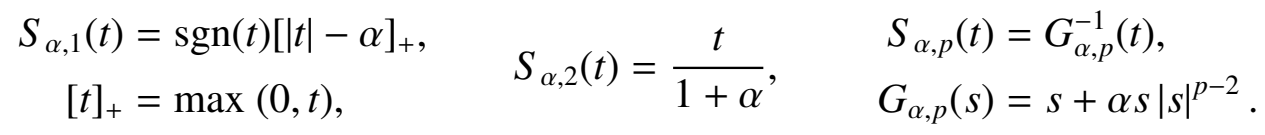


In [26], the following worst-case rate for ISTA

$$
r_{k} \leq \frac{\|A\|^{2}\left\|x_{0}-x_{\alpha}^{\delta}\right\|^{2}}{2 k}, \quad k \geq 1
$$

has been established, hence, Assumption 2.4 is satisfied with $\rho=1$. Moreover, it was shown that $\left\{r_{k}\right\}$ is a non-increasing sequence [1]. In some situations, the iterative softthresholding procedure admits a faster convergence rate, for instance, if A satisfies the finite basis injectivity property, then we have q-linear convergence [30], i.e.,

$$
r_{k} \leq C q^{k}
$$

for some $C>0$ and $q \in] 0,1[$. In this case, the descent property in Assumption 2.4 is satisfied for any $\rho>0$.

Now, examine the requirements on $\tilde{\psi}_{\alpha}$ in Assumption 2.2. For this purpose, we derive a bound on the solutions as well as the iterates. Due to the fact that $\left\{r_{k}\right\}$ for ISTA is non-increasing, it follows that

$$
\alpha\left\|x_{k}\right\|_{p}^{p} \leq T_{\alpha}^{\delta}\left(x_{k}\right) \leq T_{\alpha}^{\delta}\left(x_{0}\right)
$$

which implies

$$
\left\|x_{k}\right\|_{\infty} \leq\left\|x_{k}\right\|_{p} \leq\left(\frac{T_{\alpha}^{\delta}\left(x_{0}\right)}{\alpha}\right)^{1 / p} .
$$

Now, if we set $M_{0}=\left(T_{\alpha}^{\delta}\left(x_{0}\right) / \alpha\right)^{1 / p}$ we may define $\varphi_{p, q}$ such that it coincides with $|\cdot|^{p}$ in $\left[-M_{0}, M_{0}\right]$ and with a $q$-th power else $(q \in(1, \infty], q \geq p)$. This gives

$$
\tilde{\psi}_{\alpha}(x)=\alpha \sum_{n=1}^{\infty} \varphi_{p, q}(x(n)), \quad \varphi_{p, q}(t)= \begin{cases}|t|^{p} & \text { for }|t| \leq M_{0} \\ \frac{p}{p M_{0}^{q-p}}\left(t^{q}+\left(\frac{q}{p}-1\right) M_{0}^{q}\right) & \text { for }|t|>M_{0}\end{cases}
$$

and we see, by construction, that each $x_{k}$, and each minimizer $x_{\alpha}^{\delta}$ satisfies

$$
T_{\alpha}^{\delta}\left(x_{k}\right)=F^{\delta}\left(x_{k}\right)+\tilde{\psi}_{\alpha}\left(x_{k}\right) \quad, \quad T_{\alpha}\left(x_{\alpha}^{\delta}\right)=\tilde{T}_{\alpha}^{\delta}\left(x_{\alpha}^{\delta}\right)
$$

meaning that (4) and, consequently, Assumption 2.2, items (ii)a, (ii)b and (ii)c are satisfied. Moreover, $\tilde{\psi}_{\alpha}$ is strongly coercive since $q>1$, hence, by the Propositions 2.5 and 2.8, $0 \leq \tilde{r}_{k} \leq D_{k}$, from which follows that the remaining requirements of Assumption 2.2 are satisfied. Finally, the Fenchel conjugate $\tilde{\psi}_{\alpha}^{*}$ can be seen to read as

$$
\begin{aligned}
\tilde{\psi}_{\alpha}^{*}(x) & =\alpha \sum_{n=1}^{\infty} \varphi_{p, q}^{*}\left(\frac{x(n)}{\alpha}\right), \\
\varphi_{p, q}^{*}(t) & = \begin{cases}(p-1)\left|\frac{t}{p}\right|^{p^{*}} & \text { if }|t| \leq p M_{0}^{p-1} \\
\frac{p}{q^{*}} M_{0}^{\frac{q-p}{q-1}}\left|\frac{t}{p}\right|^{q^{*}}+\left(\frac{p}{q}-1\right) M_{0}^{p} & \text { if }|t|>p M_{0}^{p-1}\end{cases}
\end{aligned}
$$


with $p^{*}$ and $q^{*}$ being the dual exponents of $p$ and $q$, respectively, i.e., in the case of $p$, $1 / p+1 / p^{*}=1$ with $p^{*}=\infty$ in case of $p=1$. For the case where $p=1$, we agree to set $|t / p|^{p^{*}}=0$ and, for the case $q=\infty$, we set $(q-p) /(q-1)=p / q=0$ in order for the above to make sense. With this, the primal-dual gap corresponds to

$$
D(x)=\sum_{n=1}^{\infty}\left(A^{*}\left(A x-y^{\delta}\right)\right)(n) x(n)+\alpha \varphi_{p, q}(x(n))+\alpha \varphi_{p, q}^{*}\left(\frac{-\left(A^{*}\left(A x-y^{\delta}\right)\right)(n)}{\alpha}\right) .
$$

Example 2.10. The problem of minimizing (6) can also be solved numerically by using a modification of Nesterov's method called fast iterative soft-thresholding algorithm (FISTA) which has been proposed in [26]. For our purposes, it is favorable to maintain monotonicity, i.e., the property that $\left\{r_{k}\right\}$ is a non-increasing sequence as in ISTA. Therefore, we utilize monotone FISTA which has been introduced and analyzed in [2]:

$$
\left\{\begin{aligned}
x_{0} & \in \ell_{p}, \bar{x}_{0}=x_{0}, t_{0}=1 \\
z_{k+1} & =\mathbf{S}_{\alpha p, p}\left[\bar{x}_{k}-\tau A^{*}\left(A \bar{x}_{k}-y^{\delta}\right)\right] \\
t_{k+1} & =\frac{1+\sqrt{1+4 t_{k}^{2}}}{2} \\
x_{k+1} & = \begin{cases}z_{k+1} & \text { if } T_{\alpha}^{\delta}\left(z_{k+1}\right) \leq T_{\alpha}^{\delta}\left(x_{k}\right) \\
x_{k} & \text { else }\end{cases} \\
\bar{x}_{k+1} & =x_{k+1}+\frac{t_{k}}{t_{k+1}}\left(z_{k+1}-x_{k+1}\right)+\frac{t_{k}-1}{t_{k+1}}\left(x_{k+1}-x_{k}\right) .
\end{aligned}\right.
$$

Again, the step-size $\tau$ has to satisfy $0<\tau<2 /\|A\|^{2}$.

Likewise, is admits the worst-case rate

$$
r_{k} \leq \frac{2\|A\|^{2}\left\|x_{0}-x_{\alpha}^{\delta}\right\|^{2}}{(k+1)^{2}}, \quad k \geq 1
$$

leading to $\rho=2$ for this method in view of the convergence speed in Assumption 2.4. The functional values are moreover non-increasing. Therefore, for the MFISTA algorithm, $\tilde{\psi}_{\alpha}$ according to (9) may also be chosen in order to construct a primal-dual gap satisfying Assumption 2.2.

\section{Discrepancy Estimates}

The main ingredient for applying a discrepancy principle for the iterates are computable estimates for the discrepancy $F^{\delta}\left(x_{\alpha}^{\delta}\right)$ in the sense, that they do not require the knowledge of $x_{\alpha}^{\delta}$. We will derive, in the following, such estimates for the iterates $\left\{x_{k}\right\}$ of the given algorithm which involve, first, the unknown (modified) functional distance $\tilde{r}_{k}$ and, later, the primaldual gap $D_{k}=D\left(x_{k}\right)$. Throughout this section, let Assumptions 2.1 and 2.2 be satisfied. 
Furthermore, let $\delta>0$ and $\alpha>0$ be fixed and recall that we assume the existence of a $y^{\delta} \in Y$ with $\left\|y^{\delta}-y^{\dagger}\right\| \leq \delta$. For notational simplicity, we therefore write $F=F^{\delta}$ as well as $F_{k}=F\left(x_{k}\right)$. In the following we will estimate the (in practice unknown) residual term $F\left(x_{\alpha}^{\delta}\right)$ from below and from above, using the known residual $F_{k}$ in a finite iteration step $k$ and the expression $\tilde{r}_{k}$. First, we will show a more general estimate. Recall for preparation that each $x_{\alpha}^{\delta}$ also minimizes $\tilde{T}_{\alpha}^{\delta}$, the solution set can be characterized as follows (also see Lemma 2.7): An $x \in \ell_{2}$ is a minimizer of the Tikhonov functional $\tilde{T}_{\alpha}^{\delta}$ if and only if

$$
-A^{*}\left(A x-y^{\delta}\right) \in \partial \tilde{\psi}_{\alpha}(x)
$$

We will need the following basic result:

Lemma 3.1. The functional distance $\tilde{r}$ according to Assumption 2.2 item (ii)c satisfies, for any $x \in \ell_{2}$,

$$
\left\|A\left(x-x_{\alpha}^{\delta}\right)\right\|^{2} \leq 2 \tilde{r}(x) .
$$

Proof. With (11), consider the difference in the values of the functional $F+\tilde{\psi}_{\alpha}$ in the points $x$ and $x_{\alpha}^{\delta}$ :

$$
\begin{aligned}
\tilde{r}(x) & =\left(F+\tilde{\psi}_{\alpha}\right)(x)-\left(F+\tilde{\psi}_{\alpha}\right)\left(x_{\alpha}^{\delta}\right) \\
& \geq F(x)-F\left(x_{\alpha}^{\delta}\right)+\left\langle-F^{\prime}\left(x_{\alpha}^{\delta}\right), x-x_{\alpha}^{\delta}\right\rangle \\
& =\frac{\left\|A x-y^{\delta}\right\|^{2}}{2}-\frac{\left\|A x_{\alpha}^{\delta}-y^{\delta}\right\|^{2}}{2}-\left\langle A^{*}\left(A x_{\alpha}^{\delta}-y^{\delta}\right), x-x_{\alpha}^{\delta}\right\rangle \\
& =\frac{1}{2}\left\langle A x+A x_{\alpha}^{\delta}-2 y, A\left(x-x_{\alpha}^{\delta}\right)\right\rangle-\left\langle A x_{\alpha}^{\delta}-y^{\delta}, A\left(x-x_{\alpha}^{\delta}\right)\right\rangle \\
& =\frac{1}{2}\left\|A\left(x-x_{\alpha}^{\delta}\right)\right\|^{2} .
\end{aligned}
$$

Proposition 3.2. The exact discrepancy $F\left(x_{\alpha}^{\delta}\right)$ can be estimated in terms of $F, \tilde{r}$ from above and below as follows

$$
\left([\sqrt{F}-\sqrt{\tilde{r}}]_{+}\right)^{2} \leq F\left(x_{\alpha}^{\delta}\right) \leq(\sqrt{F}+\sqrt{\tilde{r}})^{2}+\tilde{r}
$$

with $[t]_{+}=\max (0, t)$.

Proof. We will make use of the following consequence of Cauchy-Schwarz's and Young's inequality: For $C>0$ and $a, b \in Y$ it holds that

$$
|\langle a, b\rangle| \leq \frac{1}{2}\left(\frac{1}{C}\|a\|^{2}+C\|b\|^{2}\right) .
$$


Now, let $x \in \ell_{2}$ be arbitrary. From the definition of $\tilde{r}$ we deduce that

$$
\begin{aligned}
F\left(x_{\alpha}^{\delta}\right) & =F(x)-\tilde{r}(x)+\tilde{\psi}_{\alpha}(x)-\tilde{\psi}_{\alpha}\left(x_{\alpha}^{\delta}\right) \\
& \stackrel{(11)}{\geq} F(x)-\tilde{r}(x)-\left\langle A x_{\alpha}^{\delta}-y^{\delta}, A\left(x-x_{\alpha}^{\delta}\right)\right\rangle \\
& \stackrel{(14)}{\geq} F(x)-\tilde{r}(x)-\frac{1}{2 C}\left\|A x_{\alpha}^{\delta}-y^{\delta}\right\|^{2}-\frac{1}{2} C\left\|A\left(x-x_{\alpha}^{\delta}\right)\right\|^{2} \\
& \stackrel{(12)}{\geq} F(x)-\tilde{r}(x)-\frac{1}{C} F\left(x_{\alpha}^{\delta}\right)-C \tilde{r}(x),
\end{aligned}
$$

and hence,

$$
\frac{C}{C+1} F(x)-C \tilde{r}(x) \leq F\left(x_{\alpha}^{\delta}\right) .
$$

If $F(x) \leq \tilde{r}(x)$, the left-hand side is non-positive, consequently, this inequality gives nothing new. In this case, we estimate by $0=\left([\sqrt{F(x)}-\sqrt{\tilde{r}(x)}]_{+}\right)^{2}$. The case where $\tilde{r}(x)=0$, it holds that $\frac{C}{C+1} F(x) \leq F\left(x_{\alpha}^{\delta}\right)$ for all $C>0$ and consequently, also for the supremum over all such $C$ implying $(\sqrt{F(x)}-\sqrt{\tilde{r}(x)})^{2}=F(x) \leq F\left(x_{\alpha}^{\delta}\right)$. In all other cases, the left-hand side is maximized by letting $C=\sqrt{F(x) / \tilde{r}(x)}-1>0$, which gives, plugged in

$$
\begin{aligned}
\frac{C}{C+1} F(x)-C \tilde{r}(x) & =\frac{\frac{F(x)}{\tilde{r}(x)}-\sqrt{\frac{F(x)}{\tilde{r}(x)}}}{\frac{F(x)}{\tilde{r}(x)}} F(x)-\sqrt{F(x) \tilde{r}(x)}+\tilde{r}(x) \\
& =F(x)-2 \sqrt{F(x) \tilde{r}(x)}+\tilde{r}(x)=(\sqrt{F(x)}-\sqrt{\tilde{r}(x)})^{2} .
\end{aligned}
$$

With (15), this yields the estimate from below.

Regarding the estimate from above, we have by the minimizing property of $x_{\alpha}^{\delta}$ and for each $x \in \ell_{2}, C>0$ :

$$
\begin{aligned}
F\left(x_{\alpha}^{\delta}\right) & \leq F(x)+\tilde{\psi}_{\alpha}(x)-\tilde{\psi}_{\alpha}\left(x_{\alpha}^{\delta}\right) \\
& \stackrel{(1)}{=} F(x)+\tilde{r}(x)+\left[F\left(x_{\alpha}^{\delta}\right)-F(x)\right] \\
& =F(x)+\tilde{r}(x)-\left[\frac{\left\|A x_{\alpha}^{\delta}-y^{\delta}\right\|^{2}}{2}-\frac{\left\|A x-y^{\delta}\right\|^{2}}{2}\right] \\
& =F(x)+\tilde{r}(x)+\left\langle\frac{A x_{\alpha}^{\delta}+A x}{2}-y^{\delta}, A\left(x_{\alpha}^{\delta}-x\right)\right\rangle \\
& =F(x)+\tilde{r}(x)+\left\langle A x-y^{\delta}, A\left(x_{\alpha}^{\delta}-x\right)\right\rangle+\frac{\left\|A\left(x_{\alpha}^{\delta}-x\right)\right\|^{2}}{2} \\
& \stackrel{(14)}{\leq} F(x)+\tilde{r}(x)+\frac{\left\|A x-y^{\delta}\right\|^{2}}{2 C}+(C+1) \frac{\left\|A\left(x-x_{\alpha}^{\delta}\right)\right\|^{2}}{2} \\
& \stackrel{(12)}{\leq} \frac{C+1}{C} F(x)+(C+2) \tilde{r}(x) .
\end{aligned}
$$


If $\tilde{r}(x)=0$, then $F\left(x_{\alpha}^{\delta}\right) \leq \frac{C+1}{C} F(x)$ for all $C>0$. This also holds for the infimum over all $C>0$, giving the desired estimate $F\left(x_{\alpha}^{\delta}\right) \leq F(x)=(\sqrt{F(x)}+\sqrt{\tilde{r}(x)})^{2}+\tilde{r}(x)$. If $\tilde{r}(x)>0$, minimization with respect to $C$ yields $C=\sqrt{F(x) / \tilde{r}(x)}$ which implies, plugged in,

$$
\begin{aligned}
F\left(x_{\alpha}^{\delta}\right) & \leq \frac{\frac{F(x)}{\tilde{r}(x)}+\sqrt{\frac{F(x)}{\tilde{r}(x)}}}{\frac{F(x)}{\tilde{r}(x)}} F(x)+\left(\sqrt{\frac{F(x)}{\tilde{r}(x)}}+2\right) \tilde{r}(x) \\
& =F(x)+2 \sqrt{F(x) \tilde{r}(x)}+2 \tilde{r}(x)=(\sqrt{F(x)}+\sqrt{\tilde{r}(x)})^{2}+\tilde{r}(x) .
\end{aligned}
$$

Corollary 3.3. It holds that

$$
\lim _{k \rightarrow \infty} F_{k}=F\left(x_{\alpha}^{\delta}\right) .
$$

If the minimization algorithm satisfies, in addition, Assumption 2.4 with convergence rate $\rho$, then the approximate discrepancies $F_{k}$ converge to $F\left(x_{\alpha}^{\delta}\right)$ with rate $\rho / 2$, i.e., there is a $C>0$ such that

$$
\left|F\left(x_{\alpha}^{\delta}\right)-F_{k}\right| \leq C k^{-\rho / 2}
$$

for all $k \geq 1$.

Proof. In the following, $C$ stands for a generic constant and may differ each times it appears. Plugging in $x_{k}$ into the estimate of $F\left(x_{\alpha}^{\delta}\right)$ from above in (13) and using the assumption $\tilde{r}_{k}=r_{k}$ for all $k \geq 1$ gives, using that $\left\{r_{k}\right\}$ as well as $F_{k}$ is bounded,

$$
F\left(x_{\alpha}^{\delta}\right)-F_{k} \leq 2 \sqrt{F_{k}} \sqrt{r_{k}}+2 r_{k} \leq C \sqrt{r_{k}} .
$$

Likewise, if $r_{k} \geq F_{k}$ it follows

$$
F_{k}-F\left(x_{\alpha}^{\delta}\right) \leq F_{k} \leq r_{k} \leq C \sqrt{r_{k}}
$$

and, if $r_{k}<F_{k}$ we have, by the estimate of $F\left(x_{\alpha}^{\delta}\right)$ from below in (13),

$$
F_{k}-F\left(x_{\alpha}^{\delta}\right) \leq 2 \sqrt{F_{k}} \sqrt{r_{k}}-r_{k} \leq C \sqrt{r_{k}}
$$

which implies that $F_{k} \rightarrow F\left(x_{\alpha}^{\delta}\right)$ since $r_{k} \rightarrow 0$ as $k \rightarrow \infty$. The claimed convergence speed follows with $\sqrt{r_{k}} \leq C k^{-\rho / 2}$.

We now like to derive a practical estimate for $F\left(x_{\alpha}^{\delta}\right)$ from (13). For this purpose, we use the primal-dual gap $D$ which satisfies $D_{k} \geq \tilde{r}_{k} \geq 0$. By monotonicity, we have

$$
\left(\left[\sqrt{F_{k}}-\sqrt{D_{k}}\right]_{+}\right)^{2} \leq F\left(x_{\alpha}^{\delta}\right) \leq\left(\sqrt{F_{k}}+\sqrt{D_{k}}\right)^{2}+D_{k}
$$

Since $\lim _{k \rightarrow \infty} F_{k}=F\left(x_{\alpha}^{\delta}\right)$ according to Corollary 3.3, we need to ensure that $D_{k}$ converges to 0 , preferably with a certain rate. 
Proposition 3.4. Let $r$ and $D$ be defined as in (1) and (3), respectively. Denote by $L\left(\tilde{\psi}_{\alpha}^{*}\right)$ the Lipschitz constant of $\tilde{\psi}_{\alpha}^{*}$ on the bounded set $M \subset \ell_{2}$ containing $\left\{-A^{*}\left(A x_{k}-y^{\delta}\right)\right\}_{k} \cup$ $\left\{-A^{*}\left(A x_{\alpha}^{\delta}-y^{\delta}\right)\right\}$.

Then, $D_{k}$ has the following behaviour:

$$
D_{k} \leq C \sqrt{r_{k}}
$$

where the constant $C$ depends on $\left\|A^{*}\right\|, L\left(\tilde{\psi}_{\alpha}^{*}\right)$ and $M$, but not on $k$.

Proof. In the following, we will use again $C$ for different constants. Observe that all the mentioned constants $C$ do not depend on the inner iteration step $k$.

First note that, due to the minimizing property of $x_{\alpha}^{\delta}$ for $T_{\alpha}^{\delta}$ and, by assumption, also for $\tilde{T}_{\alpha}^{\delta}$, we have $-A^{*}\left(A x_{\alpha}^{\delta}-y^{\delta}\right) \in \partial \tilde{\psi}_{\alpha}\left(x_{\alpha}^{\delta}\right)$ and the Fenchel identity (see, for instance, [23]) yields

$$
\left\langle-A^{*}\left(A x_{\alpha}^{\delta}-y^{\delta}\right), x\right\rangle=\tilde{\psi}_{\alpha}\left(x_{\alpha}^{\delta}\right)+\tilde{\psi}_{\alpha}^{*}\left(-A^{*}\left(A x_{\alpha}^{\delta}-y^{\delta}\right)\right)
$$

meaning $D\left(x_{\alpha}^{\delta}\right)=0$. Consequently,

$$
\begin{aligned}
D_{k} & =D_{k}-D\left(x_{\alpha}^{\delta}\right) \\
& \leq\left|\left\langle A^{*}\left(A x_{k}-y^{\delta}\right), x_{k}\right\rangle+\tilde{\psi}_{\alpha}\left(x_{k}\right)-\left\langle A^{*}\left(A x_{\alpha}^{\delta}-y^{\delta}\right), x_{\alpha}^{\delta}\right\rangle-\tilde{\psi}_{\alpha}\left(x_{\alpha}^{\delta}\right)\right| \\
& +\left|\tilde{\psi}_{\alpha}^{*}\left(-A^{*}\left(A x_{k}-y^{\delta}\right)\right)-\tilde{\psi}_{\alpha}^{*}\left(-A^{*}\left(A x_{\alpha}^{\delta}-y^{\delta}\right)\right)\right| .
\end{aligned}
$$

With (12) we yield

$$
\left\|\left(A x_{k}-y^{\delta}\right)-\left(A x_{\alpha}^{\delta}-y^{\delta}\right)\right\|=\left\|A\left(x_{k}-x_{\alpha}^{\delta}\right)\right\| \leq 2 \sqrt{r_{k}} .
$$

From the continuity of $A^{*}$ and the Lipschitz continuity of $\psi_{\alpha}^{*}$ on $\left\{-A^{*}\left(A x_{k}-y^{\delta}\right)\right\}_{k} \cup$ $\left\{-A^{*}\left(A x_{\alpha}^{\delta}-y^{\delta}\right)\right\}$ it follows that

$$
\begin{aligned}
\mid \tilde{\psi}_{\alpha}^{*}\left(-A^{*}\left(A x_{k}-y^{\delta}\right)\right) & -\tilde{\psi}_{\alpha}^{*}\left(-A^{*}\left(A x_{\alpha}^{\delta}-y^{\delta}\right)\right) \mid \\
& \leq L\left(\tilde{\psi}_{\alpha}^{*}\right)\left\|A^{*}\left(A x_{k}-y^{\delta}\right)-A^{*}\left(A x_{\alpha}^{\delta}-y^{\delta}\right)\right\| \\
& \leq L\left(\tilde{\psi}_{\alpha}^{*}\right)\left\|A^{*}\right\|\left\|\left(A x_{k}-y^{\delta}\right)-\left(A x_{\alpha}^{\delta}-y^{\delta}\right)\right\| \\
& \leq C \sqrt{r_{k}} .
\end{aligned}
$$

In order to estimate the remaining parts of (18), consider

$$
\begin{aligned}
\left|\left\langle A^{*}\left(A x_{k}-y^{\delta}\right), x_{k}\right\rangle+\tilde{\psi}_{\alpha}\left(x_{k}\right)-\left\langle A^{*}\left(A x_{\alpha}^{\delta}-y^{\delta}\right), x_{\alpha}^{\delta}\right\rangle-\tilde{\psi}_{\alpha}\left(x_{\alpha}^{\delta}\right)\right| \\
=\mid \frac{1}{2}\left\|A x_{k}-y^{\delta}\right\|^{2}+\tilde{\psi}_{\alpha}\left(x_{k}\right)-\left(\frac{1}{2}\left\|A x_{\alpha}^{\delta}-y^{\delta}\right\|^{2}+\tilde{\psi}_{\alpha}\left(x_{\alpha}^{\delta}\right)\right) \\
\quad+\frac{1}{2}\left\langle A x_{k}-y^{\delta}, A x_{k}+y^{\delta}\right\rangle-\frac{1}{2}\left\langle A x_{\alpha}^{\delta}-y^{\delta}, A x_{\alpha}^{\delta}+y^{\delta}\right\rangle \mid \\
\leq \tilde{r}_{k}+\frac{1}{2}\left|\left\|A x_{k}\right\|^{2}-\left\|y^{\delta}\right\|^{2}-\left\|A x_{\alpha}^{\delta}\right\|^{2}+\left\|y^{\delta}\right\|^{2}\right| \\
=r_{k}+\frac{1}{2}\left|\left\langle A\left(x_{k}-x_{\alpha}^{\delta}\right), A\left(x_{k}+x_{\alpha}^{\delta}\right)\right\rangle\right| \leq r_{k}+\frac{\left\|A\left(x_{k}-x_{\alpha}^{\delta}\right)\right\|\left\|A\left(x_{k}+x_{\alpha}^{\delta}\right)\right\|}{2}
\end{aligned}
$$


With the fact that $\left\|A\left(x_{k}-x_{\alpha}^{\delta}\right)\right\| \leq \sqrt{2 r_{k}}$, see Lemma 3.1, and that $\left\|A\left(x_{k}+x_{\alpha}^{\delta}\right)\right\|$ is bounded with respect to $k$, we obtain

$$
\begin{aligned}
\mid\left\langle A^{*}\left(A x_{k}-y^{\delta}\right), x_{k}\right\rangle & +\tilde{\psi}_{\alpha}\left(x_{k}\right)-\left\langle A^{*}\left(A x_{\alpha}^{\delta}-y^{\delta}\right), x_{\alpha}^{\delta}\right\rangle-\tilde{\psi}_{\alpha}\left(x_{\alpha}^{\delta}\right) \mid \\
& \leq r_{k}+C \sqrt{r_{k}} \leq C \sqrt{r_{k}} .
\end{aligned}
$$

Combining (20) and (21) completes the proof.

Corollary 3.5. The difference between the upper and lower bound in (16) converges to zero, i.e.,

$$
\lim _{k \rightarrow \infty}\left(\sqrt{F_{k}}+\sqrt{D_{k}}\right)^{2}+D_{k}-\left(\left[\sqrt{F_{k}}-\sqrt{D_{k}}\right]_{+}\right)^{2}=0 .
$$

For minimization algorithms satisfying Assumption 2.4 with convergence rate $\rho$, the convergence speed is $O\left(k^{-\rho / 4}\right)$, i.e.,

$$
\left|\left(\sqrt{F_{k}}+\sqrt{D_{k}}\right)^{2}+D_{k}-\left(\left[\sqrt{F_{k}}-\sqrt{D_{k}}\right]_{+}\right)^{2}\right| \leq C k^{-\rho / 4}
$$

for some $C>0$ not depending on $k$.

Proof. If $D_{k} \geq F_{k}$, then

$$
\left(\sqrt{F_{k}}+\sqrt{D_{k}}\right)^{2}+D_{k}-\left(\left[\sqrt{F_{k}}-\sqrt{D_{k}}\right]_{+}\right)^{2} \leq\left(2 \sqrt{D_{k}}\right)^{2}+D_{k}=5 D_{k} \leq C \sqrt{r_{k}} \leq C r_{k}^{1 / 4} .
$$

Otherwise, $D_{k}<F_{k}$, hence

$$
\left(\sqrt{F_{k}}+\sqrt{D_{k}}\right)^{2}+D_{k}-\left(\left[\sqrt{F_{k}}-\sqrt{D_{k}}\right]_{+}\right)^{2}=4 \sqrt{F_{k}} \sqrt{D_{k}}+D_{k} \leq C \sqrt{D_{k}} \leq C r_{k}^{1 / 4} .
$$

This implies the convergence as well as the rate since $r_{k}^{1 / 4} \leq C k^{-\rho / 4}$ by assumption.

\section{A Regularizing Discrepancy Principle}

We are going to choose a regularization parameter $\alpha$ from some positive decreasing sequence $\left(\alpha_{n}\right)_{n \geq 0}$ with $\alpha_{n} \rightarrow 0$ as $n \rightarrow \infty$. Usually, a geometric sequence $\alpha_{n}=\kappa^{n} \alpha_{0}, 0<\kappa<1, \alpha_{0}>0$, is considered.

Throughout this section, we require Assumptions 2.1 and 2.2 to be fulfilled, in particular, we have, for each noise level $\delta>0$ and regularization parameter $\alpha>0$ a minimization algorithm $\mathcal{P}^{\alpha, \delta}=\left\{\mathcal{P}_{1}^{\alpha, \delta}, \mathcal{P}_{2}^{\alpha, \delta}, \ldots\right\}$ for minimizing $T_{\alpha}^{\delta}$ numerically. The main idea will be to define:

Definition 4.1 (Truncated Minimization Iteration with Decreasing Regularization Parameters).

$$
\begin{aligned}
& x_{0,0}=0, \\
& x_{n, k}=\mathcal{P}_{k}^{\alpha_{n}, \delta}\left(x_{n, 0}\right), \quad 0 \leq n \leq n_{*}, \quad 1 \leq k \leq k_{n}, \\
& x_{n, 0}=x_{n-1, k_{n-1}}, \quad 1 \leq n<n_{*} .
\end{aligned}
$$


Now, we will specify how the truncation index $k_{n}$ for $n \geq 1$ can be estimated by a kind of discrepancy principle, and how the index $n_{*}=n_{*}(\delta)$ and the regularization parameter value $\alpha(\delta)=\alpha_{n_{*}}$ can be obtained for some $\delta>0$.

For simplicity, we will denote by $F_{n, k}$ the discrepancy value $F\left(x_{n, k}\right)=F^{\delta}\left(y_{n, k}\right)$ and by $D_{n, k}$ the primal-dual gap $D\left(x_{n, k}\right)$ (the latter also depending on $\alpha_{n}$ and $\delta$ ).

Definition 4.2 (Discrepancy Principle).

- Fix some $\sigma>0, \tau>1$. If $\left\|y^{\delta}\right\| \leq \sqrt{\tau} \delta$, set $x(\delta)=0, \alpha(\delta)=\delta$. Stop the algorithm.

- From now on consider the case $\left\|y^{\delta}\right\|>\sqrt{\tau} \delta$. Let $\alpha_{0}$ be chosen such that

$$
\sqrt{2 \tilde{\psi}_{\alpha_{0}}^{*}\left(A^{*} y^{\delta}\right)}<\left\|y^{\delta}\right\|-\sqrt{\tau} \delta
$$

- Set $n \leftarrow 0, k \leftarrow 0$ and initialize the iteration as in (22).

- Check the refinement criterion

$$
\left(\left[\sqrt{F_{n, k}}-\sqrt{D_{n, k}}\right]_{+}\right)^{2}>\frac{\tau \delta^{2}}{2}
$$

- If condition (26) holds, set $k_{n}=k$, the initial step for the next regularization parameter $\alpha_{n+1}$ as in (24), $n \leftarrow n+1, k \leftarrow 0$.

- Otherwise, if condition (26) does not hold, check the stopping criterion

$$
\left(\sqrt{F_{n, k}}+\sqrt{D_{n, k}}\right)^{2}+D_{n, k} \leq \frac{(1+\sigma) \tau \delta^{2}}{2} .
$$

- If (27) is not true, set $k \leftarrow k+1$, i.e., iterate (23) with the same $\alpha_{n}$. Otherwise stop the iteration, set $n_{*}=n, k_{*}=k_{n_{*}}, \alpha(\delta)=\alpha_{n_{*}}$ and $x(\delta)=x_{n_{*}, k_{*}}$.

Let us shortly discuss whether the discrepancy principle according to (26) and (27) is welldefined.

Remark 4.3 (Well-Definition of Truncated Iterative Minimization). We start with the simple observation that for any fixed $n \geq 0$ the exact discrepancy $F\left(x_{\alpha_{n}}^{\delta}\right)$ always satisfies at least one of the estimates:

- $F\left(x_{\alpha_{n}}^{\delta}\right)>\frac{\tau \delta^{2}}{2}$,

- $F\left(x_{\alpha_{n}}^{\delta}\right) \leq \frac{(1+\sigma) \tau \delta^{2}}{2}$.

The bounds (16) on the exact discrepancy approach the latter as $k \rightarrow \infty$, once

$$
D_{n, k} \geq r_{n, k} \quad \text { and } \quad D_{n, k} \rightarrow 0 \quad \text { as } \quad k \rightarrow \infty
$$

is satisfied. Indeed, this is the case since $D_{n, k} \geq \tilde{r}_{n, k}$ by Proposition 2.8, $\tilde{r}_{n, k}=r_{n, k}$ by Assumption 2.2 and $D_{n, k} \rightarrow 0$ as $k \rightarrow \infty$ by Corollary 3.5. We conclude that there will be always a $k \geq 0$ such that either (26) or (27) holds for a fixed $n$. The relaxation parameter $\sigma$ is introduced to deal with the particular situation $F\left(x_{\alpha_{n}}^{\delta}\right)=\frac{\tau \delta^{2}}{2}$. If we had $\sigma=0$, the estimate (26) would guarantee no further refinement, whereas the upper bound on $F\left(x_{\alpha_{n}}^{\delta}\right)$ would possibly never reach $\tau \delta^{2} / 2$ in (27) and consequently, the algorithm would not terminate. 
Note that it is not clear if it is possible to choose $\alpha_{0}$ according to (25). This is addressed in the following lemma.

Lemma 4.4. For each $z \in \ell_{2}$, it holds that

$$
\lim _{\alpha \rightarrow \infty} \tilde{\psi}_{\alpha}^{*}(z)=0 .
$$

Proof. First note that from $\tilde{\psi}_{\alpha}(0)=0$, see Assumption 2.2 item (ii)a, follows that $\tilde{\psi}_{\alpha}^{*}(z) \geq 0$ for each $z \in \ell_{2}$. Furthermore, as $\tilde{\psi}_{\alpha} \geq \alpha \phi$ (again Assumption 2.2 item (ii)a), the Fenchel dual satisfies

$$
\tilde{\psi}_{\alpha}^{*}(z) \leq \sup _{x \in \ell_{2}}\langle z, x\rangle-\alpha \phi(x)=\alpha \phi^{*}\left(\frac{z}{\alpha}\right) .
$$

Therefore, it is sufficient to examine the behavior of $\phi^{*}$. We first show that $\phi^{*}$ is continuous in 0 . For this purpose, we claim that there exists a $\varepsilon>0$ and a $R>0$ such that $\phi(x) \geq \varepsilon\|x\|$ for all $\|x\| \geq R$. Assume the opposite, which implies that there is, for each $n \geq 1$, a $x_{n}$ such that $\left\|x_{n}\right\| \geq n$ and $\phi\left(x_{n}\right) \leq\left\|x_{n}\right\| / n$. Defining $\tilde{x}_{n}=n x_{n} /\left\|x_{n}\right\|$ gives, since $\phi$ is convex,

$$
\phi\left(\tilde{x}_{n}\right) \leq\left(1-\frac{n}{\left\|x_{n}\right\|}\right) \phi(0)+\frac{n}{\left\|x_{n}\right\|} \phi\left(x_{n}\right) \leq 1 .
$$

From coercivity of $\phi$ now follows that $\left\{\tilde{x}_{n}\right\}$ is bounded which is a contradiction.

Hence, there exists $\varepsilon>0$ and $R>0$ such that $\phi(x) \geq \varepsilon\|x\|$ for each $\|x\| \geq R$. Choosing $z \in \ell_{2}$ such that $\|z\| \leq \varepsilon$ yields, for $\|x\| \geq R,\langle z, x\rangle-\phi(x) \leq 0$ hence

$$
\phi^{*}(z)=\sup _{\|x\| \leq R}\langle z, x\rangle-\phi(x) \leq R\|z\|,
$$

from which follows that $\phi$ is bounded from above in a neighborhood of 0 . For a convex function, this already implies continuity in 0 [24]. Note that in particular, $\phi^{*}(0)=0$.

Next, we see that $\partial \phi^{*}(0)=\{0\}$. But this is immediate since $\partial \phi^{*}(0)$ can be expressed in terms of the Fenchel equality [23]:

$$
x \in \partial \phi^{*}(0) \quad \Leftrightarrow \quad\langle 0, x\rangle=\phi(x)+\phi^{*}(0) \quad \Leftrightarrow \quad \phi(x)=0
$$

where the latter is equivalent to $x=0$ by Assumption 2.1. Hence, $\partial \phi^{*}(0)$ is a singleton and consequently, $\phi^{*}$ is Gâteaux-differentiable in 0 with vanishing derivative [24]. Therefore, for a fixed $z \in \ell_{2}$ and each $\varepsilon>0$, there exists a $\delta>0$ such that

$$
\left|\phi^{*}(t z)\right| \leq \varepsilon|t| \quad \text { for each } \quad|t|<\delta .
$$

Choosing $\alpha>\delta^{-1}$ then implies $\left|\alpha \phi^{*}(z / \alpha)\right| \leq \varepsilon$. Thus, we have shown that $\lim _{\alpha \rightarrow \infty} \alpha \phi^{*}(z / \alpha)=$ 0 which implies the claimed statement taking the above considerations into account.

As the right-hand side in (25) does not depend on $\alpha_{0}$, Lemma 4.4 shows in particular that (25) can be fulfilled if one chooses $\alpha_{0}$ large enough. In particular, the refinement criterion (26) will be fulfilled as we will see in the following lemma. This ensures, in turn, that $\alpha$ has to be refined at least once which is important for the appropriate application of the proposed discrepancy principle, as we will see in Lemma 4.8. 
Lemma 4.5. Let the data $y^{\delta}$ satisfy

$$
\left\|y^{\delta}\right\|>\sqrt{\tau} \delta .
$$

If $\alpha_{0}$ satisfies (25) then the refinement in (26) will occur for $x_{0,0}$.

Proof. For $x_{0,0}=0$ with (3) we obtain

$$
\left[\sqrt{F_{0,0}}-\sqrt{D_{0,0}}\right]_{+}=\left[\sqrt{\frac{1}{2}}\left\|y^{\delta}\right\|-\sqrt{\tilde{\psi}_{\alpha_{0}}^{*}\left(A^{*} y^{\delta}\right)}\right]_{+} .
$$

From (25) we get

$$
\sqrt{\frac{1}{2}}\left\|y^{\delta}\right\|-\sqrt{\tilde{\psi}_{\alpha_{0}}^{*}\left(A^{*} y^{\delta}\right)}>\sqrt{\frac{\tau}{2}} \delta
$$

which yields (26) for the chosen $\alpha_{0}$ and $x_{0,0}$.

We are now addressing the question whether this method constitutes a regularization method, i.e., we like to show convergence of truncated iterates $x(\delta)=x_{n_{*}, k_{*}}$ to a minimum- $\phi$-solution $x^{\dagger}$ as $\delta \rightarrow 0$. In fact, under mild assumptions, this can be established. The plan for proving this consists basically of two steps: First, we show the convergence of a related sequence of exact minimizers of $T_{\alpha}^{\delta}$ using the classical discrepancy principle of Morozov. This serves as a basis for the second step in which the regularization property for the inexact minimizers is shown.

We begin with citing existing results on Morozov discrepancy principle.

Proposition 4.6. Fix $\tau_{1}, \tau_{2}$ such that $1<\tau_{1} \leq \tau_{2}$. Let for $\delta>0$ the parameter $\alpha=\alpha(\delta)$ be chosen by the Morozov discrepancy principle

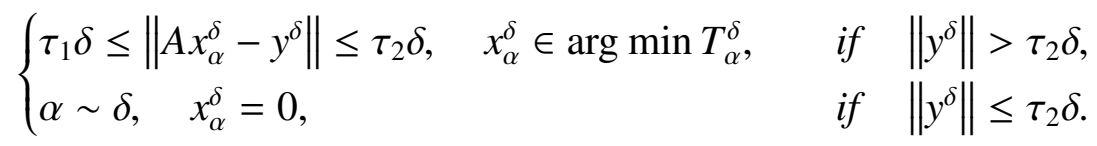

Then, $\alpha=\alpha(\delta)$ satisfies

$$
\alpha(\delta) \rightarrow 0 \quad \text { and } \quad \frac{\delta^{2}}{\alpha(\delta)} \rightarrow 0 \quad \text { as } \quad \delta \rightarrow 0
$$

Proof. Observe that if $y^{\dagger} \neq 0$, then $\tau_{2} \delta<\left\|y^{\delta}\right\|$ whenever $0<\delta<\frac{\left\|y^{\dagger}\right\|}{\left(\tau_{2}+1\right)}$. Having this in mind, one can apply the classical results about Morozov's discrepancy principle, e.g., in [3, 4], minding that Assumption 2.1 yields the necessary prerequisites. If $y^{\dagger}=0$, then $\tau_{2} \delta<\left\|y^{\delta}\right\|$ is always violated and the parameter choice $\alpha(\delta) \sim \delta$ yields the desired result.

Remark 4.7. Proposition 4.6 shows the typical asymptotic behaviour of the parameter value $\alpha(\delta)$, which is sometimes assumed to hold a-priori to get the convergence of a regularization method. In the a-priori case, strong convergence of the exact minimizers has been shown [1] for the weighted $\ell_{p}$-norms with positive weights bounded away from zero. 
For general penalties, the Morozov discrepancy principle (D) only guarantees weak convergence [4]. To show strong convergence of the regularized solutions with respect to some general penalty, an additional assumption

$$
x_{n} \rightarrow \bar{x}, \phi\left(x_{n}\right) \rightarrow \phi(\bar{x})<\infty \quad \Longrightarrow \quad \phi\left(x_{n}-\bar{x}\right) \rightarrow 0 .
$$

on the penalty term is required [4].

If the $\phi$-minimizing solution $x^{\dagger}$ is not unique, the regularized solutions converge (weakly or strongly) to the set $S^{\dagger}$ of the generalized solutions. The uniqueness of the generalized solution $x^{\dagger}$ is guaranteed either in the case of strictly convex $\phi$, or, if $\phi$ is only convex, in the case $\operatorname{ker}(A)=\{0\}$.

In the following lemma we make use of Definition 4.2 to find a parameter $\gamma$ close to $\alpha(\delta)=\alpha_{n_{*}}$, such that each corresponding exact minimizer $x_{\gamma}^{\delta}$ satisfies the classical discrepancy principle of Morozov (D).

Lemma 4.8. Let $\delta>0$ be fixed and let $y^{\delta}$ be such that $\left\|y^{\delta}\right\|>\sqrt{\tau} \delta$. Let $\alpha(\delta)=\alpha_{n_{*}}$ be found by the discrepancy principle described in Definition 4.2. Then there exists a $\gamma=\gamma(\delta) \in\left[\alpha_{n_{*}}, \alpha_{n_{*}-1}\right]$, where $\alpha_{n_{*}-1}$ is the penultimate parameter value, such that

$$
\frac{\tau \delta^{2}}{2} \leq F\left(x_{\gamma}^{\delta}\right) \leq(1+\sigma) \frac{\tau \delta^{2}}{2}
$$

where $x_{\gamma}^{\delta}$ is a minimizer of $T_{\gamma}^{\delta}$.

Proof. Note that Lemma 4.5 assures that $n_{*} \geq 1$, i.e. there exists $\alpha_{n_{*}-1}$.

From Definition 4.2 it follows that

$$
F\left(x_{\alpha_{n_{*}-1}}^{\delta}\right) \geq \frac{\tau \delta^{2}}{2}
$$

and

$$
F\left(x_{\alpha_{*}}^{\delta}\right) \leq \frac{(1+\sigma) \tau \delta^{2}}{2} .
$$

In fact, the estimate (31) is a consequence of the refinement inequality (26), which holds for $n=n_{*}-1$ and $k=k_{n_{*}-1}$, and the estimate from below for $F\left(x_{\alpha_{n_{*}-1}}\right)$ in (16). The estimate (32) is obtained due to the stopping criterion (27), which holds for $n=n_{*}$, and the estimate from above for the exact discrepancy $F\left(x_{\alpha_{n *}}^{\delta}\right)$ in (16).

Denote by $f(\alpha):=F\left(x_{\alpha}^{\delta}\right)$. It is a known fact that $f$ is well-defined (since single-valued for each $\alpha>0$ ) and a monotonically increasing continuous function [31, Section 2.7]. Therefore and due to (31) and (32), the intersection $\Sigma=\left[f\left(\alpha_{n_{*}}\right), f\left(\alpha_{n_{*}-1}\right)\right] \cap\left[\frac{\tau \delta^{2}}{2}, \frac{(1+\sigma) \tau \delta^{2}}{2}\right]$ is nonempty. As $\left[f\left(\alpha_{n_{*}}\right), f\left(\alpha_{n_{*}-1}\right)\right]=f\left(\left[\alpha_{n_{*}}, \alpha_{n_{*}-1}\right]\right)$, an arbitrary $\gamma=f^{-1}(\xi)$ for $\xi \in \Sigma$ satisfies $\gamma \in\left[\alpha_{n_{*}}, \alpha_{n_{*}-1}\right]$ as well as $F\left(x_{\alpha_{n_{*}}}^{\delta}\right) \leq F\left(x_{\gamma}^{\delta}\right) \leq F\left(x_{\alpha_{n_{*}-1}}^{\delta}\right)$, consequently, (30) holds. 
Remark 4.9. Inequality (30) assures that $x_{\gamma}^{\delta}$ satisfies the classical discrepancy principle (D) with $\tau_{1}=\sqrt{\tau}$ and $\tau_{2}=\sqrt{(1+\sigma) \tau}$.

In the following we show that the parameter values $\alpha(\delta)$, chosen by Definition 4.2 exhibit the classical decay as $\delta \rightarrow 0$.

Lemma 4.10. Let for some $\delta>0, \alpha(\delta)$ be a parameter value found by Definition 4.2. Then, for $\delta \rightarrow 0, \alpha(\delta)$ satisfies

$$
\alpha(\delta) \rightarrow 0 \quad \text { and } \quad \frac{\delta^{2}}{\alpha(\delta)} \rightarrow 0
$$

Proof. First, assume that $y^{\dagger} \neq 0$. Then, for sufficiently small $\delta$ we are in the situation of Lemma 4.8, i.e. $\left\|y^{\delta}\right\|>\sqrt{\tau} \delta$ holds. Now, according to Proposition 4.6, each choice $\gamma(\delta)$ such that

$$
\sqrt{\tau} \delta \leq\left\|A x_{\gamma}^{\delta}-y^{\delta}\right\| \leq \sqrt{(1+\sigma) \tau} \delta
$$

satisfies

$$
\gamma(\delta) \rightarrow 0 \quad \text { and } \quad \frac{\delta^{2}}{\gamma(\delta)} \rightarrow 0
$$

as $\delta \rightarrow 0$. Now, according to Lemma 4.8 we have $\gamma(\delta) \in\left[\alpha(\delta), \kappa^{-1} \alpha(\delta)\right]$ for a particular choice $\gamma(\delta)$. Consequently,

$$
\alpha(\delta) \leq \gamma(\delta) \rightarrow 0, \quad \frac{\delta^{2}}{\alpha(\delta)} \leq \frac{\delta^{2}}{\kappa \gamma(\delta)} \rightarrow 0,
$$

i.e. (33) holds. If $y^{\dagger}=0$, then $\left\|y^{\delta}\right\| \leq \sqrt{\tau} \delta$ and consequently, $\alpha(\delta)=\delta$ by Definition 4.2, which immediately yields (33).

In order to show convergence of the approximate solutions, we will need some properties of the penalty evaluated in the exact minimizers $x_{\alpha(\delta)}^{\delta}$, where $\alpha(\delta)$ is chosen by Definition 4.2.

Lemma 4.11. Let, for $\delta>0$, the parameter values $\alpha=\alpha(\delta)$ be given by Definition 4.2 and $x_{\alpha}^{\delta}$ be the corresponding "exact" regularizing solution, i.e. either $x_{\alpha}^{\delta}=0$ if $\left\|y^{\delta}\right\| \leq \sqrt{\tau} \delta$, or $x_{\alpha}^{\delta} \in \arg \min T_{\alpha}^{\delta}$ if $\left\|y^{\delta}\right\|>\sqrt{\tau} \delta$, respectively. Then, for sufficiently small values of $\delta$ the penalty values $\phi\left(x_{\alpha}^{\delta}\right)$ satisfy

$$
\phi\left(x_{\alpha}^{\delta}\right) \leq \frac{\delta^{2}}{2 \alpha}+\phi\left(x^{\dagger}\right)
$$

Moreover, $x_{\alpha}^{\delta} \rightarrow \mathcal{S}^{\dagger}$ in the sense that each subsequence admits a subsequence weakly converging to an element in $\mathcal{S}^{\dagger}$. Finally,

$$
\phi\left(x_{\alpha}^{\delta}\right) \rightarrow \phi\left(x^{\dagger}\right) \quad \text { as } \quad \delta \rightarrow 0
$$

Proof. The proof follows the lines of [4, Lem. 4.1, Cor. 4.2]. Let $\left\{\delta_{i}\right\}$ be an arbitrary sequence with $\delta_{i} \rightarrow 0$ as $i \rightarrow \infty$. Denote by $\alpha_{i}=\alpha\left(\delta_{i}\right)$ as well as $x_{i}=x_{\alpha_{i}}^{\delta_{i}}$. First, consider the case 
$y^{\dagger} \neq 0$. Then, for a sufficiently large $i_{0}$ and all $i \geq i_{0},\left\|y^{\delta_{i}}\right\|>\sqrt{\tau} \delta_{i}$ holds. From the minimizing property of $x_{i}$ we obtain

$$
\alpha_{i} \phi\left(x_{i}\right) \leq \frac{1}{2}\left\|A x_{i}-y^{\delta_{i}}\right\|^{2}+\alpha_{i} \phi\left(x_{i}\right) \leq \frac{\delta_{i}^{2}}{2}+\alpha_{i} \phi\left(x^{\dagger}\right)
$$

whereas we get (34) and with (33)

$$
\limsup _{i \rightarrow \infty} \phi\left(x_{i}\right) \leq \phi\left(x^{\dagger}\right)
$$

i.a., the sequence $\phi\left(x_{i}\right)$ is bounded. From the estimate (32) we know that

$$
\left\|A x_{i}-y^{\delta_{i}}\right\| \rightarrow 0 \quad \text { as } \quad i \rightarrow \infty
$$

Due to the boundedness of $\phi\left(x_{\alpha_{i}}^{\delta_{i}}\right)$ and coercivity of $\phi$ we can extract a weakly converging subsequence $x_{i^{\prime}}:=x_{\alpha_{i^{\prime}}}^{\delta_{i^{\prime}}} \rightarrow \bar{x}$. With the weak lower semi-continuity of $\left\|A \cdot-y^{\dagger}\right\|$ we obtain

$$
\left\|A \bar{x}-y^{\dagger}\right\| \leq \liminf _{i^{\prime} \rightarrow \infty}\left\|A x_{i^{\prime}}-y^{\dagger}\right\| \leq \liminf _{i^{\prime} \rightarrow \infty}\left(\left\|A x_{i^{\prime}}-y^{\delta_{i^{\prime}}}\right\|+\delta_{i^{\prime}}\right)=0
$$

which means that $\bar{x}$ is a solution of $A x=y^{\dagger}$. Moreover, since $\phi$ is lower semi-continuous, we get

$$
\phi(\bar{x}) \leq \liminf _{i^{\prime} \rightarrow \infty} \phi\left(x_{i^{\prime}}\right) \leq \limsup _{i^{\prime} \rightarrow \infty} \phi\left(x_{i^{\prime}}\right) \leq \phi\left(x^{\dagger}\right) .
$$

From the assumption that $x^{\dagger}$ is a $\phi$-minimizing solution, we get $\phi(\bar{x})=\phi\left(x^{\dagger}\right)$ and $\phi\left(x_{i^{\prime}}\right) \rightarrow$ $\phi\left(x^{\dagger}\right)$. Since the argument applies to any subsequence of $x_{\alpha_{i}}^{\delta_{i}}$ of exact minimizers, we conclude (35) and the weak convergence of the whole sequence towards the set $\mathcal{S}^{\dagger}$.

Now, consider the case $y^{\dagger}=0$. Then, for sufficiently small $\delta>0,\left\|y^{\delta}\right\| \leq \sqrt{\tau} \delta$ holds. By assumption, we obtain $x_{\alpha}^{\delta}=0$, which automatically satisfies (34). Since 0 is a $\phi$-minimizing solution in the case $y^{\dagger}=0$, and $\phi(0)=0$, we trivially obtain (35).

Now we are going to show the main result of this paper, the regularization property of the inexact minimizers, obtained by the truncated minimization algorithm with adaptively chosen regularization parameters.

Theorem 4.12. Let Assumptions 2.1 and 2.2 be satisfied. Let $\alpha(\delta)$ and $x(\delta)$ be given by Definitions 4.1 and 4.2 for some $\delta>0$. Then, for $\delta \rightarrow 0$, the penalty values converge towards $\phi\left(x^{\dagger}\right)$, where $x^{\dagger}$ is a $\phi$-minimizing solution of $A x=y^{\dagger}$ :

$$
\phi(x(\delta)) \rightarrow \phi\left(x^{\dagger}\right)
$$

Moreover, the inexact minimizers $x(\delta)$ converge weakly towards the set $\mathcal{S}^{\dagger}$ of $\phi$-minimizing solutions of $A x=y^{\dagger}$. 
Proof. Consider a sequence $\delta_{i} \rightarrow 0$. Denote again by $\alpha_{i}=\alpha\left(\delta_{i}\right)$ the corresponding parameter value and by $x_{i}=x_{\alpha_{i}}^{\delta_{i}}, \tilde{x}_{i}=x\left(\delta_{i}\right)$ the exact and the inexact regularized solutions, respectively. The exact regularized solutions $x_{i}$ are chosen as in Lemma 4.11.

First, consider the case $y^{\dagger} \neq 0$ and assume $\delta_{i}$ to be small enough, such that $\left\|y^{\delta}\right\|>\sqrt{\tau} \delta$ holds. We are going to show that

$$
\phi\left(\tilde{x}_{i}\right) \rightarrow \phi\left(x^{\dagger}\right) \quad \text { as } \quad i \rightarrow \infty
$$

The triangle inequality yields

$$
\left|\phi\left(\tilde{x}_{i}\right)-\phi\left(x^{\dagger}\right)\right| \leq\left|\phi\left(\tilde{x}_{i}\right)-\phi\left(x_{i}\right)\right|+\left|\phi\left(x_{i}\right)-\phi\left(x^{\dagger}\right)\right|
$$

for which the second term on the right hand side tends to zero by (35). Thus, let us estimate the term $\left|\phi\left(\tilde{x}_{i}\right)-\phi\left(x_{i}\right)\right|$. For this purpose, we are going to estimate the discrepancy error $\left|F\left(x_{i}\right)-F\left(\tilde{x}_{i}\right)\right|$.

We are essentially using the estimate of the exact discrepancy (16) and Definition 4.2. The estimate from above in (16) on $F\left(x_{i}\right)$ yields

$$
\sqrt{F\left(\tilde{x}_{i}\right)}-\sqrt{D\left(\tilde{x}_{i}\right)} \leq\left[\sqrt{F\left(\tilde{x}_{i}\right)}-\sqrt{D\left(\tilde{x}_{i}\right)}\right]_{+} \leq \sqrt{F\left(x_{i}\right)},
$$

and therefore

$$
\sqrt{F\left(\tilde{x}_{i}\right)}-\sqrt{F\left(x_{i}\right)} \leq \sqrt{D\left(\tilde{x}_{i}\right)} .
$$

Moreover, from the stopping criterion (27) and from the upper bound in (16) on $F\left(x_{i}\right)$ we obtain

$$
F\left(\tilde{x}_{i}\right) \leq \frac{(1+\sigma) \tau \delta_{i}^{2}}{2}, \quad F\left(x_{i}\right) \leq \frac{(1+\sigma) \tau \delta_{i}^{2}}{2} \quad \text { and } \quad D\left(\tilde{x}_{i}\right) \leq \frac{(1+\sigma) \tau \delta_{i}^{2}}{2}
$$

We deduce for

$$
\begin{aligned}
F\left(\tilde{x}_{i}\right)-F\left(x_{i}\right) & \leq\left(\sqrt{F\left(\tilde{x}_{i}\right)}-\sqrt{F\left(x_{i}\right)}\right)\left(\sqrt{F\left(\tilde{x}_{i}\right)}+\sqrt{F\left(x_{i}\right)}\right) \\
& \stackrel{(36),(37)}{\leq} \sqrt{D\left(\tilde{x}_{i}\right)} \cdot 2 \sqrt{\frac{(1+\sigma) \tau \delta_{i}^{2}}{2}} \\
& \stackrel{(37)}{\leq} \sqrt{\frac{(1+\sigma) \tau \delta_{i}^{2}}{2}} \cdot 2 \sqrt{\frac{(1+\sigma) \tau \delta_{i}^{2}}{2}}=(1+\sigma) \tau \delta_{i}^{2} .
\end{aligned}
$$

On the other hand, from the upper bound in (16) on $F\left(x_{i}\right)$ we directly obtain

$$
\begin{aligned}
F\left(x_{i}\right)-F\left(\tilde{x}_{i}\right) & \leq 2 D\left(\tilde{x}_{i}\right)+2 \sqrt{F\left(\tilde{x}_{i}\right)} \sqrt{D\left(\tilde{x}_{i}\right)} \\
& \stackrel{(37)}{\leq} 2 \frac{(1+\sigma) \tau \delta_{i}^{2}}{2}+2 \sqrt{\frac{(1+\sigma) \tau \delta_{i}^{2}}{2}} \sqrt{\frac{(1+\sigma) \tau \delta_{i}^{2}}{2}} \\
& =2(1+\sigma) \tau \delta_{i}^{2},
\end{aligned}
$$


which, together with (38), yields

$$
\left|F\left(x_{i}\right)-F\left(\tilde{x}_{i}\right)\right| \leq 2(1+\sigma) \tau \delta_{i}^{2} .
$$

Consider now

$$
\begin{aligned}
& \left|\tilde{\psi}_{\alpha_{i}}\left(\tilde{x}_{i}\right)-\tilde{\psi}_{\alpha_{i}}\left(x_{i}\right)\right| \stackrel{(1),(2)}{=}\left|\tilde{r}_{\alpha_{i}}\left(\tilde{x}_{i}\right)-F\left(\tilde{x}_{i}\right)+F\left(x_{i}\right)\right| \\
& \stackrel{(5)}{\leq} \quad D\left(\tilde{x}_{i}\right)+\left|F\left(x_{i}\right)-F\left(\tilde{x}_{i}\right)\right| \\
& \stackrel{\text { (37),(40) }}{\leq} \frac{(1+\sigma) \tau \delta_{i}^{2}}{2}+2(1+\sigma) \tau \delta_{i}^{2} \\
& \leq \quad \frac{5}{2}(1+\sigma) \tau \delta_{i}^{2} .
\end{aligned}
$$

Now, from $r_{k}=\tilde{r}_{k}$ in Assumption 2.2 item (ii)c follows $\phi\left(\tilde{x}_{i}\right)-\phi\left(x_{i}\right)=\frac{1}{\alpha_{i}}\left(\tilde{\psi}_{\alpha_{i}}\left(\tilde{x}_{i}\right)-\tilde{\psi}_{\alpha_{i}}\left(x_{i}\right)\right)$, thus

$$
\left|\phi\left(\tilde{x}_{i}\right)-\phi\left(x_{i}\right)\right| \leq \frac{5}{2}(1+\sigma) \frac{\tau \delta_{i}^{2}}{\alpha_{i}},
$$

which tends to zero as $i \rightarrow \infty$ by Proposition 4.6. With Lemma 4.11, which assures that $\phi\left(x_{i}\right) \rightarrow \phi\left(x^{\dagger}\right)$, this yields

$$
\phi\left(\tilde{x}_{i}\right) \rightarrow \phi\left(x^{\dagger}\right) \quad \text { as } \quad i \rightarrow \infty .
$$

It follows that the sequence $\left\{\phi\left(\tilde{x}_{i}\right)\right\}$ is bounded. The coercivity of $\phi$ implies that $\left\{\tilde{x}_{i}\right\}$ is bounded in $\ell_{2}$. Hence, there exists a weakly convergent subsequence $\tilde{x}_{i^{\prime}} \rightarrow \bar{x}$. From the weak lower semi-continuity of $\left\|A \cdot-y^{\dagger}\right\|$ and (27) we get again

$$
\begin{aligned}
\left\|A \bar{x}-y^{\dagger}\right\| & \leq \liminf _{i^{\prime} \rightarrow \infty}\left\|A \tilde{x}_{i^{\prime}}-y^{\dagger}\right\| \leq \liminf _{i^{\prime} \rightarrow \infty}\left(\left\|A \tilde{x}_{i^{\prime}}-y^{\delta_{i^{\prime}}}\right\|+\delta_{i^{\prime}}\right) \\
& \stackrel{(27)}{\leq} \liminf _{i^{\prime} \rightarrow \infty}\left[((1+\sigma) \tau)^{1 / 2} \delta_{i^{\prime}}+\delta_{i^{\prime}}\right]=0,
\end{aligned}
$$

i.e. $\bar{x}$ is an exact solution of $A x=y^{\dagger}$. As still $\phi\left(\tilde{x}_{i^{\prime}}\right) \rightarrow \phi\left(x^{\dagger}\right)$ as $i \rightarrow \infty$, the weak lower semi-continuity of $\phi$ implies that

$$
\phi(\bar{x}) \leq \liminf _{i^{\prime} \rightarrow \infty} \phi\left(\tilde{x}_{i^{\prime}}\right)=\phi\left(x^{\dagger}\right) .
$$

Therefore, $\bar{x}$ is also a $\phi$-minimizing solution of $A x=y^{\dagger}$. Since the above argument applies to any subsequence of $\left\{\tilde{x}_{i}\right\}$, we conclude weak convergence of the whole sequence $\left\{\tilde{x}_{i}\right\}$ to the set $\mathcal{S}^{\dagger}$, i.e., all weak limit points $\bar{x}$ of $\tilde{x}_{i}$ are elements from $\mathcal{S}^{\dagger}$. Moreover, since the value $\phi(\bar{x})$ is the same for all $\bar{x} \in \mathcal{S}^{\dagger}$, we get $\phi\left(\tilde{x}_{i}\right) \rightarrow \phi(\bar{x})=\phi\left(x^{\dagger}\right)$ as $i \rightarrow \infty$.

In the case $y^{\dagger}=0$, we get, for sufficiently small $\delta>0, x(\delta)=0$ by definition and $0 \in \mathcal{S}^{\dagger}$. Thus, the convergence of the penalty values and the weak convergence towards the set of generalized solutions is trivially satisfied. 
Remark 4.13. As a side product we can obtain from the proof that the iteration according to Definition 4.1 with parameter choice according to Definition 4.2 yields, for each $\delta>0$ an approximate solution $x(\delta)$ and parameter $\alpha(\delta)$ such that

$$
\left|\phi(x(\delta))-\phi\left(x_{\alpha(\delta)}^{\delta}\right)\right| \leq \frac{5(1+\sigma) \tau}{2} \frac{\delta^{2}}{\alpha(\delta)}
$$

where $x_{\alpha(\delta)}^{\delta}=0$ if $\left\|y^{\delta}\right\| \leq \sqrt{\tau} \delta$ and $x_{\alpha(\delta)}^{\delta} \in \arg \min T_{\alpha(\delta)}^{\delta}$ if $\left\|y^{\delta}\right\|>\sqrt{\tau} \delta$.

Finally, if the $\phi$-minimizing solution is unique, cf. Remark 4.7, under an additional condition on $\phi$, strong convergence with respect to penalty follows.

Corollary 4.14. Let $x^{\dagger}$ be the unique $\phi$-minimizing solution of $A x=y^{\dagger}$. Let the assumptions of Theorem 4.12 be satisfied. Additionally, let condition (29) hold. Then the regularized solutions $x(\delta)$ converge to $x^{\dagger}$ with respect to $\phi$ :

$$
\phi\left(x(\delta)-x^{\dagger}\right) \rightarrow 0 \quad \text { as } \quad \delta \rightarrow 0 .
$$

Proof. In case of unique $\phi$-minimizing solution $x^{\dagger}$ we get from Theorem 4.12 the weak convergence $x(\delta) \rightarrow x^{\dagger}$ as $\delta \rightarrow 0$. With $\phi(x(\delta)) \rightarrow \phi\left(x^{\dagger}\right)$ and condition (29) we deduce $\phi\left(x(\delta)-x^{\dagger}\right) \rightarrow 0$ as $\delta \rightarrow 0$.

Note that the condition (29) is satisfied e.g. by the classical $\ell_{p}$-penalty term [32].

\section{Convergence Rates}

In this section, we discuss convergence rates for the proposed methods under some additional assumptions on the penalty functional $\phi$, the operator $A$ and, of course, a source condition. Analogously to Section 4, our argumentation is based on properties of the Tikhonov regularization with a-priori parameter choice which are then extended to the a-posteriori case. Within this framework, Morozov's classical discrepancy principle is addressed as a side product before obtaining a rate for the regularizing discrepancy principle of Section 4.

Let us recall some techniques for deriving convergence rates. Typically, a smoothness assumption on the solution is required to obtain a quantitative information about the regularization error. A well-known source condition in the Hilbert space setting is $x^{\dagger} \in$ $\mathrm{R}\left(\left(A^{*} A\right)^{\mu}\right)$, which covers a range of smoothness conditions. In Banach spaces, due to the lack of a spectral representation, only particular cases such as $\psi^{\prime}\left(x^{\dagger}\right) \in \mathrm{R}\left(A^{*}\right)$ or $\psi^{\prime}\left(x^{\dagger}\right) \in \mathrm{R}\left(A^{*} J A\right)$, where $J$ is a duality mapping between $Y$ and $Y^{*}$, have been considered $[16,18]$. This rigid assumption can be weakened by introducing an approximative source condition, as proposed in [19] for the low rate cases, which correspond to $\mu<1 / 2$ in the Hilbert space setting. There, optimal rates have been obtained under an approximative source condition. In the following, we adapt the strategy of [1], where the source condition combines two independent 
assumptions on the smoothness of the solution and the smoothing property of the operator, which was first introduced for regularization in Hilbert scales [22].

We are going to extend the result of [1, Prop. 4.5], developed for the a-priori case to more general penalty functionals $\phi$ as well as to the approximate regularization with a-posteriori parameter choice rule proposed in Section 4. We will require some additional properties of the penalty functional $\phi$ :

Assumption 5.1. Consider the penalty functional $\phi$ from Assumption 2.1. We assume that

(i) for any $\varrho>0$ the sets $\mathcal{B}_{\varrho}=\{x \in X: \phi(x) \leq \varrho\}$ are compact in the solution space $X=\ell_{2}$ and $\mathcal{B}_{\varrho} \cap \operatorname{ker}(A)=\{0\}$;

(ii) $\phi$ is even, i.e., $\phi(-x)=\phi(x)$ for all $x \in \ell_{2}$,

(iii) $\phi$ satisfies the quasi-triangle inequality, i.e. there exists $Q \geq 1$ such that for any $x, x^{\prime} \in \ell_{2}$ it holds that

$$
\phi\left(x+x^{\prime}\right) \leq Q\left(\phi(x)+\phi\left(x^{\prime}\right)\right) .
$$

Remark 5.2. For the special case $\phi(x)=\|x\|_{w, p}^{p}=\sum_{\lambda} w_{\lambda}\left|x_{\lambda}\right|^{p}, 1 \leq p \leq 2$, the sets $\mathcal{B}_{\varrho}, \varrho>0$ are compact in $\ell_{2}$ if the positive weights $\left\{w_{\lambda}\right\}$ tend to infinity in the sense that

$$
\forall C>0 \quad \sharp\left\{\lambda \in \Lambda: w_{\lambda} \leq C\right\}<\infty,
$$

see [1]. Moreover, $\phi(x)=\|x\|_{w, p}^{p}$ satisfies the quasi-triangle inequality with $Q=2^{p-1}$.

We are going to utilize the following source condition.

Assumption 5.3. Suppose that an exact solution $x^{\dagger} \in \mathcal{S}^{\dagger}$ satisfies the constraint

$$
\phi\left(x^{\dagger}\right) \leq \varrho
$$

for some given $\varrho>0$.

Let us discuss the motivation behind Assumptions 5.1 and 5.3. If the source condition Assumption 5.3 is satisfied and we also know that $y^{\delta}$ lies within a distance $\delta$ of $A x^{\dagger}$ in $Y$ (which is part of Assumption 2.1), then the exact solution $x^{\dagger}$ belongs to the set

$$
\mathcal{F}(\delta, \varrho)=\left\{x \in X:\left\|A x-y^{\delta}\right\| \leq \delta, \phi(x) \leq \varrho\right\} .
$$

To measure the quality of approximation of the exact solution $x^{\dagger}$ with respect to any $x \in$ $\mathcal{F}(\delta, \varrho)$, it is convenient to introduce the modulus of continuity of $A^{-1}$ which is given by

$$
M_{t}(\delta, \varrho)=\sup \{\|z\|:\|A z\| \leq \delta, \phi(z) \leq \varrho\}
$$

for fixed $\delta>0$ and $\rho>0$. Here, $z$ represents the approximation error $x-x^{\dagger}$.

In fact, due to the linearity of $A$ and $\phi$ being even and satisfying the quasi-triangle inequality (which is the case when Assumption 5.1 is satisfied), the diameter of $\mathcal{F}(\delta, \varrho)$, which estimates for any $\tilde{x} \in \mathcal{F}(\delta, \varrho)$ the distance to the exact solution $x^{\dagger}$, can be bounded by $M_{t}(2 \delta, 2 Q \varrho)$ as

$$
\begin{aligned}
\sup \left\{\left\|x-x^{\prime}\right\|:\left\|A x-y^{\delta}\right\| \leq \delta, \phi(x) \leq\right. & \left.\leq,\left\|A x^{\prime}-y^{\delta}\right\| \leq \delta, \phi\left(x^{\prime}\right) \leq \varrho\right\} \\
& \leq \sup \left\{\left\|x-x^{\prime}\right\|:\left\|A\left(x-x^{\prime}\right)\right\| \leq 2 \delta, \phi\left(x-x^{\prime}\right) \leq 2 Q \varrho\right\} .
\end{aligned}
$$


Remark 5.4. Let Assumption 5.1 be fulfilled. Then we can show that the value $M_{t}(\delta, \varrho)$ is well-defined. In fact, from $\mathcal{B}_{\varrho} \cap \operatorname{ker}(A)=\{0\}$ we infer that the linear map $A: \mathcal{B}_{\varrho} \rightarrow A\left(\mathcal{B}_{\varrho}\right)$ is injective and onto, and therefore has an inverse, which coincides with $A^{\dagger}: A\left(\mathcal{B}_{\varrho}\right) \rightarrow \mathcal{B}_{\varrho}$. Moreover, since $A$ is continuous, and $\mathcal{B}_{\varrho}$ is assumed to be compact, the inverse is also continuous, and the value $M_{t}(\delta, \varrho)$ is bounded for a fixed $\delta$ and tends to zero as $\delta \rightarrow 0$. For more details on the modulus of continuity see [33, Section 3.2].

In order to describe convergence rates for regularized solutions, we are interested in the worstcase error of a regularization method with respect to "true solutions" $x$ satisfying the source condition and all possible data $y^{\delta}$ within a $\delta$-distance to the exact data $y^{\dagger}=A x$. This modulus of convergence, reads, in the case of Tikhonov regularization, as follows:

$$
\begin{aligned}
M_{v}^{\mathrm{Tik}}(\alpha, \delta, \varrho)=\sup \left\{\left\|x_{\alpha}^{\delta}-x\right\|: x \in \ell_{2}, \phi(x) \leq \varrho, y^{\delta} \in Y,\right. & \\
& \left.\left\|A x-y^{\delta}\right\| \leq \delta, x_{\alpha}^{\delta} \in \arg \min T_{\alpha}^{\delta}\right\} .
\end{aligned}
$$

Our aim is to obtain an a-priori parameter choice $\alpha(\delta)$ such that $M_{v}^{\mathrm{Tik}}(\alpha(\delta), \delta, \varrho)$ vanishes with a certain rate as $\delta \rightarrow 0$. Likewise, we like to derive the convergence behavior of the moduli of convergence for the Morozov discrepancy principle and the inexact discrepancy principle of Definitions 4.1 and 4.2 which read as follows:

$$
\begin{gathered}
M_{v}^{\mathrm{Mor}}(\delta, \varrho)=\sup \left\{\left\|x_{\alpha}^{\delta}-x\right\|: x \in \ell_{2}, \phi(x) \leq \varrho, y^{\delta} \in Y,\left\|A x-y^{\delta}\right\| \leq \delta,\right. \\
\left.x_{\alpha}^{\delta} \text { chosen according to (D) }\right\},
\end{gathered}
$$

where the bounds in (D) satisfy $1<\tau_{1}<\tau_{2}$ and

$$
\begin{aligned}
& M_{v}^{\mathrm{iMor}}(\delta, \varrho)=\sup \left\{\|x(\delta)-x\|: x \in \ell_{2}, \phi(x) \leq \varrho, y^{\delta} \in Y,\left\|A x-y^{\delta}\right\| \leq \delta,\right. \\
& x(\delta) \text { according to Definitions } 4.1 \text { and } 4.2\} \text {. }
\end{aligned}
$$

First, we obtain bounds for the modulus of convergence for exact Tikhonov minimizers and motivate an a-priori parameter choice which is optimal with respect to these bounds. The following proposition generalizes the result of [1, Prop. 4.5] to a larger class of $\phi$.

Proposition 5.5. Let $\phi$ satisfy Assumption 2.1. Then, for $\alpha>0$ and $\delta>0$, the modulus of convergence according to (47) obeys

$$
M_{t}(\delta, \varrho) \leq M_{v}^{\mathrm{Tik}}(\alpha, \delta, \varrho) \leq M_{t}\left(\delta+\delta^{\prime}, Q\left(\varrho+\varrho^{\prime}\right)\right)
$$

where

$$
\delta^{\prime}=\sqrt{\delta^{2}+2 \alpha \varrho}, \quad \varrho^{\prime}=\varrho+\frac{\delta^{2}}{2 \alpha} .
$$

Proof. The proof essentially follows the lines of [1, Prop. 4.5]. However, since we take a general penalty $\phi$ instead of the weighted $\ell_{p}$-norm, and in order to highlight the difference with the a-posteriori case, we would like to recall the main idea. Let $x \in \ell_{2}, \phi(x) \leq \varrho$ and 
$y^{\delta} \in Y$ such that $\left\|A x-y^{\delta}\right\| \leq \delta$. In particular, $x \in \mathcal{F}(\delta, \varrho)$. Moreover, let $x_{\alpha}^{\delta}$ be a minimizer of the associated Tikhonov functional $T_{\alpha}^{\delta}$. From the minimization property of $x_{\alpha}^{\delta}, \phi \geq 0$ and the above facts for $x$ and $y^{\delta}$, we get

$$
\frac{\left\|A x_{\alpha}^{\delta}-y^{\delta}\right\|^{2}}{2} \leq T_{\alpha}^{\delta}\left(x_{\alpha}^{\delta}\right) \leq T_{\alpha}^{\delta}(x) \leq \frac{\delta^{2}}{2}+\alpha \varrho .
$$

Moreover, the same can be used to estimate

$$
\alpha \phi\left(x_{\alpha}^{\delta}\right) \leq T_{\alpha}^{\delta}\left(x_{\alpha}^{\delta}\right) \leq \frac{\delta^{2}}{2}+\alpha \varrho .
$$

Consequently, $x_{\alpha}^{\delta} \in \mathcal{F}\left(\delta^{\prime}, \varrho^{\prime}\right)$ with $\delta^{\prime}$ and $\varrho^{\prime}$ given in (51). In particular, it follows that $\left\|A\left(x_{\alpha}^{\delta}-x\right)\right\| \leq \delta+\delta^{\prime}$ and $\phi\left(x_{\alpha}^{\delta}-x\right) \leq Q\left(\varrho+\varrho^{\prime}\right)$. Hence, remembering (46),

$$
\left\|x_{\alpha}^{\delta}-x\right\| \leq M_{t}\left(\delta+\delta^{\prime}, Q\left(\varrho+\varrho^{\prime}\right)\right)
$$

which implies the estimate from above. To obtain the lower bound, observe that for $y^{\delta}=0$, $x_{\alpha}^{\delta}=0$ is the unique minimizer of $T_{\alpha}^{\delta}$ as $\phi(x)=0$ if and only if $x=0$ (confer Assumption 2.1). Hence, for each $x \in \ell_{2}, \phi(x) \leq \varrho$ and $\|A x\| \leq \delta$ we also have $\left\|A x-y^{\delta}\right\| \leq \delta,\|x\|=\left\|x_{\alpha}^{\delta}-x\right\|$ and consequently,

$$
\|x\| \leq M_{v}^{\mathrm{Tik}}(\alpha, \delta, \varrho)
$$

by (47) which implies, by (46), the desired result.

We now like to make the estimate (50) as tight as possible with respect to its asymptotic decay as $\delta \rightarrow 0$. This will become clear if one can derive an expression for $M_{t}(\delta, \varrho)$ in terms of $\delta$ and $\varrho$. In the following example, we are going to combine the source condition (45) with an additional regularity assumption on the operator $A$ in order to use the explicit expression for $M_{t}(\delta, \varrho)$ from [1, Prop. 4.7].

Example 5.6. Assume that $\ell_{2}$ is the coefficient space with respect to a wavelet basis and that

$$
\phi(x)=\|x\|_{w, p}^{p}=\sum_{\lambda} w_{\lambda}\left|x_{\lambda}\right|^{p}, \quad 1 \leq p \leq 2
$$

where the weights are $w_{\lambda}=2^{\zeta p|\lambda|}$ with $|\lambda|$ denoting the scale component of the wavelet index and $\zeta \in \mathbb{R}$. It is known [34] that the sequence spaces $\ell_{w, p}$ can be identified with the Besov spaces $B_{p, p}^{s}\left(\mathbb{R}^{d}\right)$, where $\zeta=s+d\left(\frac{1}{2}-\frac{1}{p}\right)$ by the equivalence

$$
\left\|\sum_{\lambda} x_{\lambda} \Psi_{\lambda}\right\|_{B_{p, p}^{s}\left(\mathbb{R}^{d}\right)} \sim\|x\|_{w, p}
$$

for an appropriate wavelet basis $\Psi=\left\{\Psi_{\lambda}\right\}$. For $\zeta>0$, it can be shown that the sets $\|\cdot\|_{w, p} \leq \varrho$, $\varrho>0$ are compact in $X=\ell_{2}$, see Remark 5.2. Moreover, $\phi$ is the p-th power of a norm and therefore satisfies Assumption 5.1, again with $Q=2^{p-1}$. 
Furthermore, we assume the operator A to be a smoothing operator of order $\eta$, i.e., that the norm equivalence

$$
\|A x\|^{2} \sim \sum_{\lambda} 2^{-2 \eta|\lambda|} x_{\lambda}^{2}
$$

holds. Typically such a condition is assumed for regularization in Hilbert scales, cf. [22]. Then, according to [1], the modulus of continuity $M_{t}(\delta, \varrho)$ is of the order

$$
M_{t}(\delta, \varrho) \sim \delta^{\frac{\zeta}{\zeta+\eta}} \varrho^{\frac{\eta}{\zeta+\eta}}
$$

Note that the rate with respect to $\delta$ depends on the smoothing properties of $A$ and becomes worse with increasing $\eta$.

As we have seen in Example 5.6, in a particular case, the modulus of continuity $M_{t}$ depends homogeneously on $\delta$ and $\rho$. Suppose that we have an a-priori parameter choice $\delta \mapsto \alpha(\delta)$. Then one can see that in the case of Example 5.6, the bounds $M_{t}(\delta, \varrho)$ and $M_{t}\left(\delta+\delta^{\prime}, Q\left(\varrho+\varrho^{\prime}\right)\right)$ for $M_{v}^{\mathrm{Tik}}(\alpha(\delta), \delta, \rho)$ obtained in (50) are of the same order with respect to $\delta$, and hence tight up to a constant, if and only if $\delta^{\prime} / \delta$ and $\rho^{\prime}$ is bounded for all $\delta>0$. In view of (51), this is equivalent to $\alpha(\delta) \varrho / \delta^{2}$ being bounded for all $\delta>0$. This yields the well-known optimal a-priori parameter choice $\alpha(\delta) \sim \frac{\delta^{2}}{\varrho}$.

In the following we derive the bounds on the modulus of convergence in case of the discrepancy principle applied to exact and inexact Tikhonov minimizers. We start with the classical Morozov discrepancy principle.

Proposition 5.7. Let $\phi$ satisfy Assumption 5.1. Then, the modulus of convergence (48) for Morozov's discrepancy principle (D) obeys

$$
M_{t}(\delta, \varrho) \leq M_{v}^{\mathrm{Mor}}(\delta, \varrho) \leq M_{t}\left(\left(\tau_{2}+1\right) \delta, 2 Q \varrho\right) .
$$

Proof. First, consider the case $\left\|y^{\delta}\right\|>\tau_{2} \delta$. Let $x \in \ell_{2}, \phi(x) \leq \rho, y^{\delta} \in Y$ with $\left\|A x-y^{\delta}\right\| \leq \delta$ and suppose that $\alpha>0$ is chosen according to (D), i.e., a minimizer $x_{\alpha}^{\delta}$ of $T_{\alpha}^{\delta}$ satisfies $\tau_{1} \delta \leq\left\|A x_{\alpha}^{\delta}-y^{\delta}\right\| \leq \tau_{2} \delta$. Then, with

$$
\frac{\delta^{2}}{2}+\alpha \phi\left(x_{\alpha}^{\delta}\right) \leq \frac{\tau_{1}^{2} \delta^{2}}{2}+\alpha \phi\left(x_{\alpha}^{\delta}\right) \leq T_{\alpha}^{\delta}\left(x_{\alpha}^{\delta}\right) \leq T_{\alpha}^{\delta}(x) \leq \frac{\delta^{2}}{2}+\alpha \phi(x),
$$

we get

$$
\phi\left(x_{\alpha}^{\delta}\right) \leq \phi(x) \leq \varrho .
$$

With the quasi-triangle inequality one then gets

$$
\phi\left(x_{\alpha}^{\delta}-x\right) \leq Q\left(\phi\left(x_{\alpha}^{\delta}\right)+\phi(x)\right) \leq 2 Q \varrho
$$

while the discrepancy principle yields

$$
\left\|A\left(x_{\alpha}^{\delta}-x\right)\right\| \leq\left\|A x_{\alpha}^{\delta}-y^{\delta}\right\|+\left\|A x-y^{\delta}\right\| \leq\left(\tau_{2}+1\right) \delta .
$$


This shows by the definition of $M_{t}\left(\left(\tau_{2}+1\right) \delta, 2 Q \varrho\right)$ in (46) that

$$
\left\|x_{\alpha}^{\delta}-x\right\| \leq M_{t}\left(\left(\tau_{2}+1\right) \delta, 2 Q \varrho\right)
$$

in case $x_{\alpha}^{\delta}$ is chosen according to Morozov's discrepancy principle.

In the case where $x \in \ell_{2}, \phi(x) \leq \rho, y^{\delta} \in Y,\left\|A x-y^{\delta}\right\| \leq \delta$ and $\left\|y^{\delta}\right\| \leq \tau_{2} \delta$, the discrepancy principle (D) yields $x_{\alpha}^{\delta}=0$ and we have that $\|A x\| \leq\left\|A x-y^{\delta}\right\|+\left\|y^{\delta}\right\| \leq\left(1+\tau_{2}\right) \delta$ as well as $\phi(x) \leq \varrho \leq 2 Q \varrho$, hence

$$
\left\|x_{\alpha}^{\delta}-x\right\|=\|x\| \leq M_{t}\left(\left(1+\tau_{2}\right) \delta, 2 Q \varrho\right) .
$$

This yields the estimate from above in (54) by the definition of $M_{v}^{\mathrm{Mor}}(\delta, \varrho)$ in (48).

For the estimate from below, let $x \in \ell_{2}$ such that $\|A x\| \leq \delta$ and $\phi(x) \leq \varrho$. Choosing $y^{\delta}=0$ and $x_{\alpha}^{\delta}=0$ we see that $\left\|A x-y^{\delta}\right\| \leq \delta$ and $\left\|y^{\delta}\right\| \leq \tau_{2} \delta$ and hence, again by (48),

$$
\|x\|=\left\|x-x_{\alpha}^{\delta}\right\| \leq M_{v}^{\mathrm{Mor}}(\delta, \varrho)
$$

which yields the result by virtue of (46).

We assume again that the modulus of continuity $M_{t}$ has the behaviour (53). Then the bounds on the modulus of convergence $M_{v}^{\text {Mor }}$ in Proposition 5.7 are optimal without any necessary assumption on the parameter behaviour. Therefore, the optimality of the rates of the exact Tikhonov regularization with discrepancy principle does not contradict with the result of Proposition 4.6. In fact, the a-priori parameter choice $\alpha \sim \delta^{2}$ is only a sufficient condition for order optimality, whereas $\frac{\delta^{2}}{\alpha} \rightarrow 0$ necessarily holds if Morozov's discrepancy principle is assumed.

Now, we are going to estimate the modulus of convergence in case of the inexact Tikhonov minimizers chosen by the approximate discrepancy principle in Definitions 4.1 and 4.2.

Proposition 5.8. Let $\phi$ satisfy assumptions of Theorem 4.12 and Assumption 5.1. Let the inexact regularization $(x(\delta), \alpha(\delta))$ be obtained by Definitions 4.1 and 4.2. Then, the corresponding modulus of convergence $M_{v}^{\mathrm{i} M o r}$ obeys

$$
M_{t}(\delta, \varrho) \leq M_{v}^{\mathrm{iMor}}(\delta, \varrho) \leq M_{t}\left(\delta+\delta^{\prime}, Q\left(\varrho+\varrho^{\prime}\right)\right) .
$$

where

$$
\delta^{\prime}=\sqrt{(1+\sigma) \tau} \delta, \quad \varrho^{\prime}=\left(\frac{5(1+\sigma) \tau+1}{\kappa(\tau-1)}+1\right) \varrho .
$$

Proof. Let $\left\|A x-y^{\delta}\right\| \leq \delta$ and let $x \in \ell_{2}$ be such that $\phi(x) \leq \varrho$ holds. First, consider the case $\left\|y^{\delta}\right\|>\sqrt{\tau} \delta$. Then the iteration in Definitions 4.1 and 4.2 will be stopped by the rule (27).

Recall that we denote by $x(\delta)$ the last iterate and by $x_{\alpha(\delta)}^{\delta}$ a minimizer of the Tikhonov functional $T_{\alpha(\delta)}^{\delta}$. By the definition of $F(x(\delta))$ and the stopping rule (27), we get

$$
\left\|A x(\delta)-y^{\delta}\right\| \leq \sqrt{(1+\sigma) \tau} \delta=\delta^{\prime} .
$$


Moreover, we obtain

$$
\begin{aligned}
\phi(x(\delta)) & \leq(x(\delta))-\phi\left(x_{\alpha(\delta)}^{\delta}\right)+\phi\left(x_{\alpha(\delta)}^{\delta}\right) \\
\stackrel{(34),(43)}{\leq} & \frac{5(1+\sigma) \tau \delta^{2}}{2 \alpha(\delta)}+\frac{\delta^{2}}{2 \alpha(\delta)}+\phi\left(x^{\dagger}\right) \\
\stackrel{(45)}{\leq} & (5(1+\sigma) \tau+1) \frac{\delta^{2}}{2 \alpha(\delta)}+\varrho .
\end{aligned}
$$

In the following, we are going to estimate the ratio $\frac{\delta^{2}}{\alpha(\delta)}$ in terms of $\varrho$. As shown in Lemma 4.8, there is a $\gamma \in[\alpha(\delta), \alpha(\delta) / \kappa]$ satisfying the classical discrepancy principle with the lower bound

$$
\frac{\tau \delta^{2}}{2} \leq F\left(x_{\gamma}^{\delta}\right)
$$

As $x_{\gamma}^{\delta}$ is a minimizer of $T_{\gamma}^{\delta}$, we obtain

$$
\frac{\tau \delta^{2}}{2}+\gamma \phi\left(x_{\gamma}^{\delta}\right) \leq F\left(x_{\gamma}^{\delta}\right)+\gamma \phi\left(x_{\gamma}^{\delta}\right) \leq F^{\delta}\left(x^{\dagger}\right)+\gamma \phi\left(x^{\dagger}\right) \leq \frac{\delta^{2}}{2}+\gamma \phi\left(x^{\dagger}\right),
$$

which yields

$$
\frac{(\tau-1) \delta^{2}}{2 \gamma} \leq \phi\left(x^{\dagger}\right)-\phi\left(x_{\gamma}^{\delta}\right) \leq \phi\left(x^{\dagger}\right) \leq \varrho .
$$

Since we know from Lemma 4.8 that $\alpha(\delta) \geq \kappa \gamma$, we finally get

$$
\frac{\delta^{2}}{\alpha(\delta)} \leq \frac{\delta^{2}}{\kappa \gamma} \leq \frac{2 \varrho}{(\tau-1) \kappa}
$$

Combining (56) and (57), we obtain

$$
\phi(x(\delta)) \leq \varrho^{\prime} .
$$

In the case $\left\|y^{\delta}\right\| \leq \sqrt{\tau} \delta$, we have $x(\delta)=0$ by Definition 4.2. Hence, we get

$$
\left\|A x(\delta)-y^{\delta}\right\| \leq \sqrt{\tau} \delta \leq \delta^{\prime}
$$

and

$$
\phi(x(\delta))=0 \leq \varrho^{\prime} .
$$

In both cases, the upper bound in (55) follows from the estimates

$$
\|A(x(\delta)-x)\| \leq\left\|A x(\delta)-y^{\delta}\right\|+\left\|A x-y^{\delta}\right\| \leq \delta^{\prime}+\delta
$$

and

$$
\phi(x(\delta)-x) \leq Q(\phi(x(\delta))+\phi(x)) \leq Q\left(\varrho^{\prime}+\varrho\right) .
$$

To show the lower bound, we take again a particular $y^{\delta}=0$. Again, our inexact minimizer is $x(\delta)=0$ by Definition 4.2 for all $\delta>0$ and we can argue in the same way as in Proposition 5.5. 
If we assume again that the modulus of continuity $M_{t}$ has the behaviour (53) we see that the lower and the upper bound for $M_{v}^{\mathrm{iMor}}$ already have the same asymptotic rates with respect to $\delta$ and $\varrho$.

Finally, under the regularity conditions (52) specified in Example 5.6, the optimal convergence rates can be given explicitly as $M_{v}^{\mathrm{iMor}}(\delta, \rho) \sim M_{t}(\delta, \rho)$ for varying $\delta$. In terms of (53) we have, consequently, the convergence rate $\delta^{\frac{\zeta}{\zeta+\eta}}$.

Corollary 5.9. Let $x^{\dagger}$ and A satisfy the source condition (45) in the situation of Example 5.6 with some $\zeta, \eta>0$. Let the regularization $\left(x_{\text {reg }}, \alpha_{\text {reg }}\right)$ be one of the following:

- an exact minimizer $x_{\alpha}^{\delta}$ of $T_{\alpha}^{\delta}$ with $\alpha(\delta)$ chosen a-priori such that $\frac{\delta^{2}}{\alpha} \sim \varrho$;

- $x_{\alpha}^{\delta}$ and $\alpha\left(\delta, y^{\delta}\right)$ chosen by the classical Morozov discrepancy principle (D);

- $x(\delta)$ with $\alpha(\delta)$ from Definition 4.1 combined with the approximate discrepancy principle, Definition 4.2.

Then, the modulus of convergence (46) for such a regularization is of the order

$$
M_{v}(\delta, \varrho) \sim \delta^{\frac{\zeta}{\zeta+\eta}} \varrho^{\frac{\eta}{\zeta+\eta}}
$$

Remark 5.10 (Saturation). Note that the convergence rates $\delta^{\frac{2 \mu}{2 \mu+1}}$ for the classical Tikhonov regularization with $\mu$-type source condition saturate at $\mu_{0}=1$ for the a-priori parameter choice rule and at $\mu_{0}=\frac{1}{2}$ for the Morozov discrepancy principle [33]. In this case, $\mu_{0}$ is called qualification of the method.

For the Tikhonov regularization in Hilbert scales [22], the saturation issue can be overcome, i.e. higher rates than for the $\mu$-type source condition can be achieved. In particular, as shown in [22, Thm. 2.3], the rates $\delta^{\frac{\zeta}{\zeta+\eta}} \varrho^{\frac{\eta}{\zeta+\eta}}$ are order optimal for any choices of $\zeta, \eta>0$, if the source condition is formulated with respect to the penalty norm $\|\cdot\|_{w, 2}, w_{\lambda}=2^{2 \zeta|\lambda|}$ with the same index $\zeta$.

More generally, if $\xi$ defines the weights of the penalty and $\zeta$ the weights of the smoothness source condition, as in Example 5.6 with $p=2$, the order optimal rates $\delta^{\frac{\zeta}{\zeta+\eta}} \varrho^{\frac{\eta}{\zeta+\eta}}$ are obtained for all $\xi, \zeta, \eta>0$ and $\mu_{0} \geq 1$ satisfying $\zeta \leq \min \left\{2 \xi+\eta, 2 \mu_{0}(\eta+\xi)\right\}$, where $\mu_{0}$ is the qualification of the regularization method with the a-priori parameter choice rule [22, Thm. 2.3]. The case $\xi=\zeta$ is covered by this result.

However, to the best of our knowledge, no generalization of the saturation issue in case of separable source condition to the Banach spaces case is available in the literature.

\section{Numerical Experiments}

Let $\Omega \subset \mathbb{R}^{2}$ be a bounded domain, $k \in L_{2}\left(\mathbb{R}^{2}\right)$ and let the convolution operator $K: L_{2}(\Omega) \rightarrow$ $L_{2}(\Omega)$ be defined as

$$
K(\boldsymbol{x})(t)=\int_{\Omega} k(t-s) \boldsymbol{x}(s) d s, \quad \boldsymbol{x} \in L_{2}(\Omega)
$$




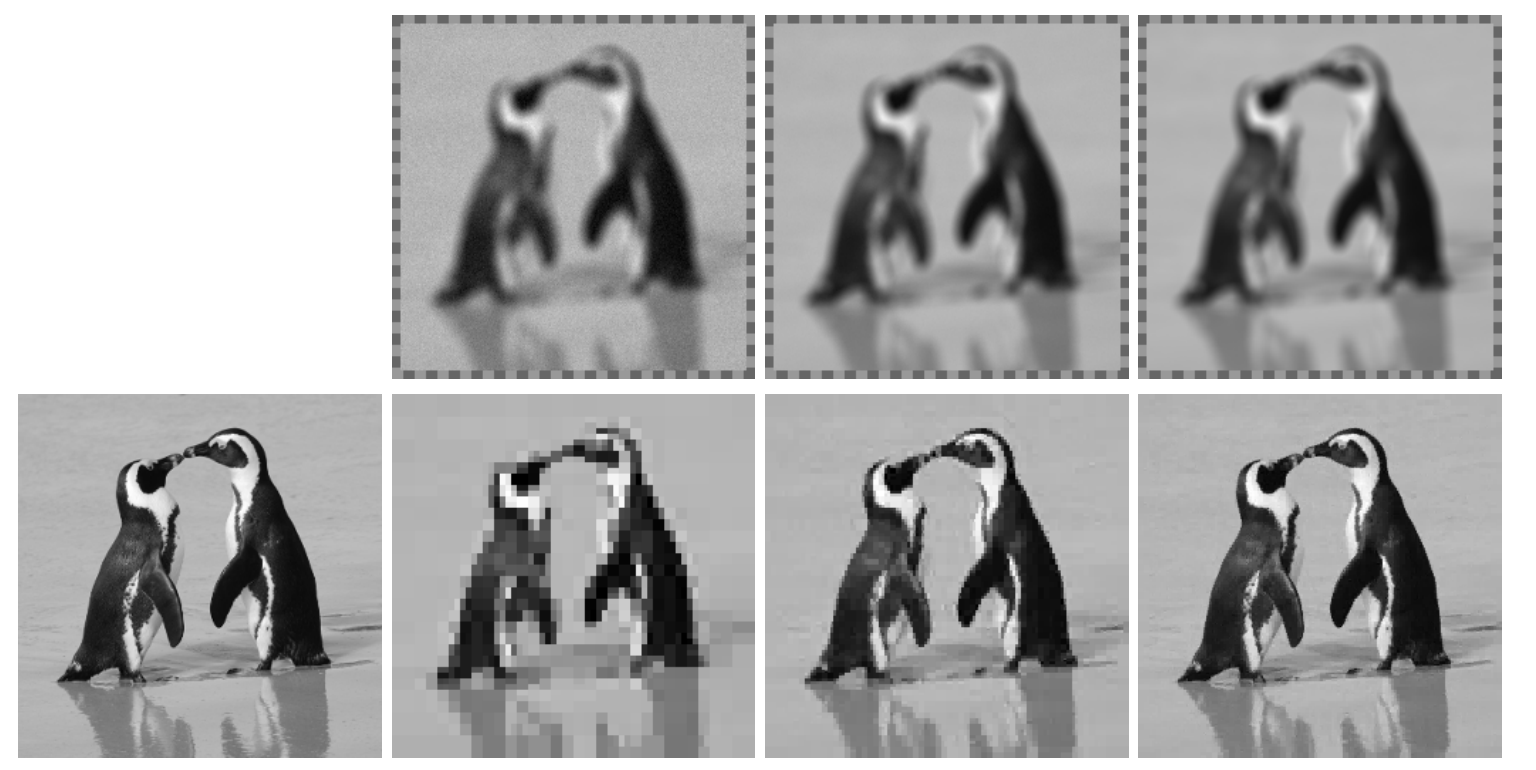

Figure 1. Reconstructions for different noise levels. Top, from left to right: Data $y^{\delta}$ for relative noise levels of $3.4 \%, 0.6 \%$ and $0.1 \%$, respectively. Bottom, from left to right: Original data $\boldsymbol{x}^{\dagger}$, MFISTA reconstructions $\boldsymbol{x}(\delta)=B x(\delta)$ from the data in the top row. (Image by Paul Mannix@Flickr [36], licenced under CC-BY-2.0, http://creativecommons.org/ licenses/by/2.0/legalcode.)

As the convolution operator (59) can be approximated by operators of finite rank, see [35, Cor II.3.3] and the subsequent example, it is compact and therefore, its inversion is ill-posed [33]. We are going to apply the discrepancy principle, Definition 4.2, to iterative methods which minimize the Tikhonov functional with $\ell_{1}$-norm regularization. To this end, we introduce an orthonormal wavelet basis $\left\{\psi_{\lambda}\right\}_{\lambda}$ of $L_{2}(\Omega)$, in this particular case we specify $\Omega=[0,1]^{2}$ and $\left\{\psi_{\lambda}\right\}_{\lambda}$ to be the Haar basis on the unit cube. We define the synthesis operator $B: \ell_{2} \rightarrow L_{2}(\Omega)$ by $B x=\sum_{\lambda} x_{\lambda} \psi_{\lambda}$. Then we can apply our approach to the operator $A$, defined as $A=K B$ and mapping the $\ell_{2}$-coefficients $x$ of some function $\boldsymbol{x}$ to the convolved function $\boldsymbol{y}=K \boldsymbol{x}$. Given some noisy data $\boldsymbol{y}^{\delta}$ with $\left\|\boldsymbol{y}^{\dagger}-\boldsymbol{y}^{\delta}\right\| \leq \delta$, we aim at solving, by Tikhonov regularization, the system

$$
A x=y^{\delta}
$$

instead of solving $K \boldsymbol{x}=\boldsymbol{y}^{\dagger}$. We therefore denote by $x^{\dagger}$ the wavelet coefficients of the exact solution $\boldsymbol{x}^{\dagger}$.

We performed deblurring experiments for a sample image with $256 \times 256$ pixels which has been convolved with a circular out-of-focus kernel of 13 pixels diameter and distorted by noise. A spatial discretization of step-size 1 has been chosen, hence $\Omega=[0,256]^{2}$ with the pixels being represented by a translated unit cubes. Finally, the range of grayscale values corresponds to the interval $[0,1]$. Note that all errors are measured with respect to this representation. Approximate solutions $x(\delta)$ according to Definitions 4.1 and 4.2 were 

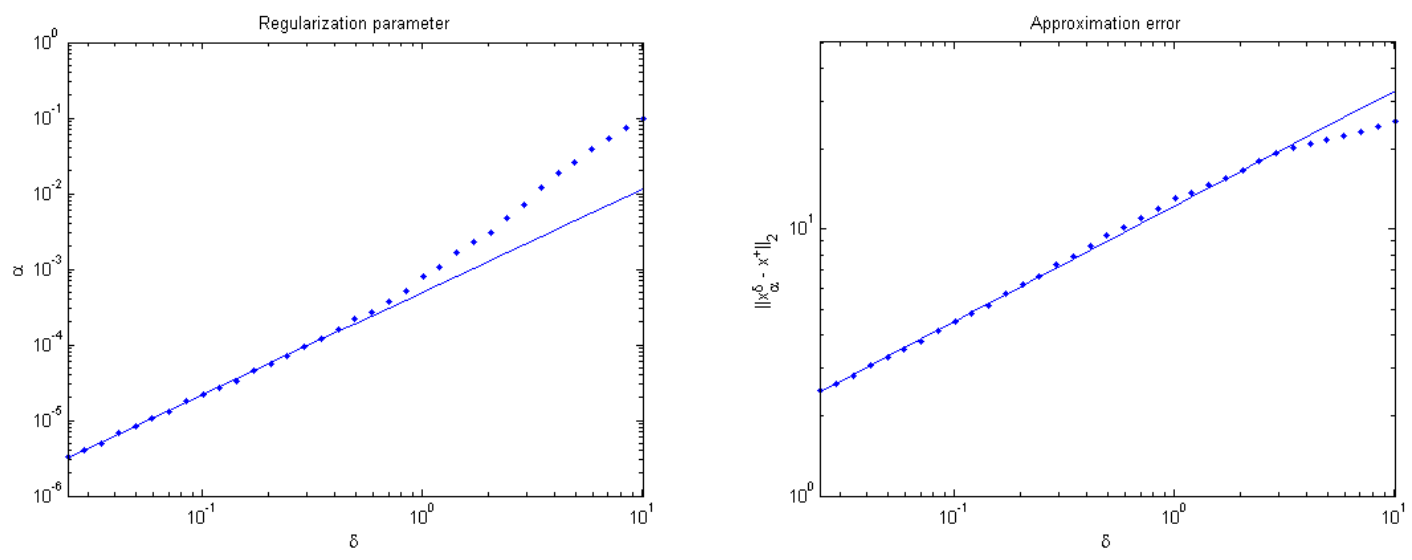

Figure 2. Left: Regularization parameter $\alpha$ versus (absolute) noise level $\delta$. Approximate rate $\alpha \approx 0.0005 \delta^{1.3596}$. Right: $\ell_{2}$-approximation error versus noise level $\delta$. Approximate rate $\left\|x(\delta)-x^{\dagger}\right\|_{\ell_{2}} \approx 12.1813 \delta^{0.4319}$. Dotted: computed values, solid: fitted values.

computed for a range of noise levels $\delta$ using the iterative soft-thresholding (ISTA) and monotone fast iterative soft-thresholding (MFISTA) iterations, see Examples 2.9 and 2.10, respectively, with parameters $\alpha_{0}=0.1, \kappa=0.9, \tau=1.1$ and $\sigma=0.25$. The outcome of the procedure in case of MFISTA for some representative noise levels $\delta$ is depicted in Figure 1. It can be seen that indeed, the procedure yields a reasonable choice of the regularization parameter as well as an iteration number such that the recovered images are sharper on the one hand and neither over- nor under-smoothed, on the other hand. We also observed that visually, the results of ISTA and MFISTA can not be distinguished, indicating that the method is also robust with respect to the concrete minimization algorithm utilized in Definition 4.1, a hypothesis whose verification can also be found at the end of this section.

Figure 2 shows the convergence behaviour, again for the MFISTA realization of the algorithm, of the approximation error $\left\|x(\delta)-x^{\dagger}\right\|_{2}$ and the regularization parameter $\alpha(\delta)=\alpha_{n_{*}}$ as $\delta \rightarrow 0$. Indeed, one can observe that regularization parameter as well as approximation error satisfies some asymptotic rates. In particular, the statement $\delta^{2} / \alpha \rightarrow 0$ of Lemma 4.10 can be confirmed. The results also indicate that there is some rate for which the approximation error tends to zero. This behaviour can be explained by the results from Section 5 (see Example 5.6 and Corollary 5.9) in infinite dimensions, although the concrete values of $\eta$ and $\zeta$ can, of course, not be obtained as by discretizing, we cut off the wavelet series after finitely many coefficients. Additionally, we performed a quantitative comparison between the ISTA iteration and the MFISTA iteration for the minimization of the respective Tikhonov functionals. The comparison admits no significant difference in approximation quality, as it can be seen in Figure 3, left. However, the computational complexity can be reduced significantly by using MFISTA, see Figure 3, right. One has to note, nevertheless, that the overall iteration number is quite high, in particular, if one wants to obtain highly accurate solutions at low noise levels. 

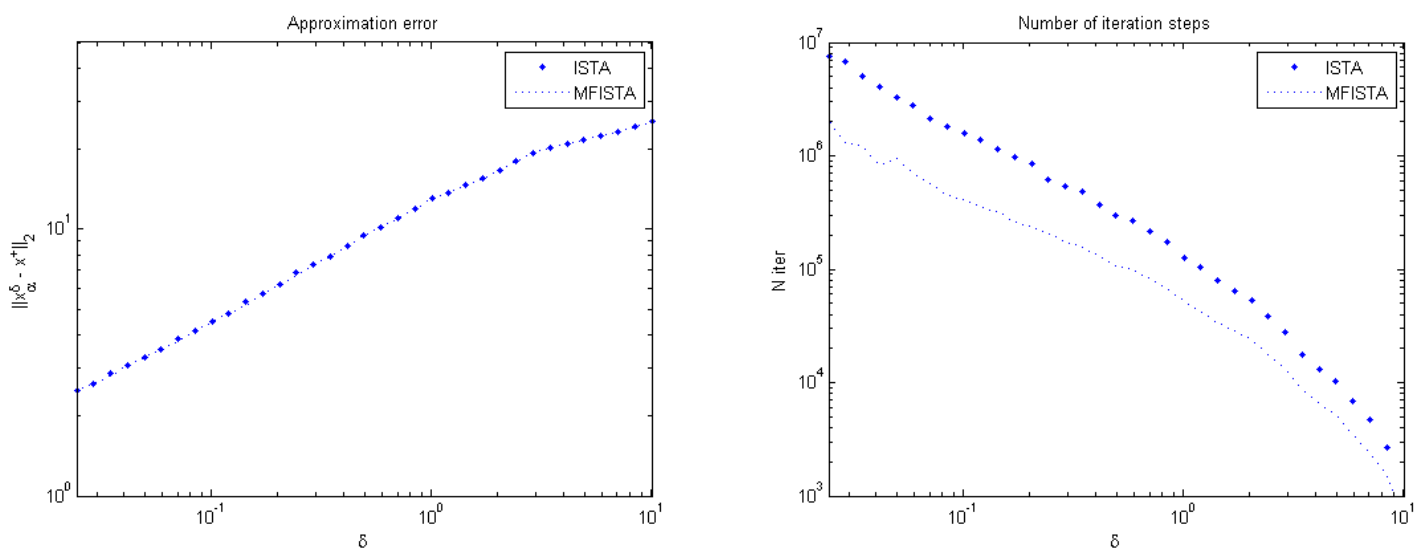

Figure 3. Comparison of the performance of the two different minimization algorithms, ISTA and MFISTA. Left: Reconstruction quality (approximation error). Right: Overall number of iteration steps.

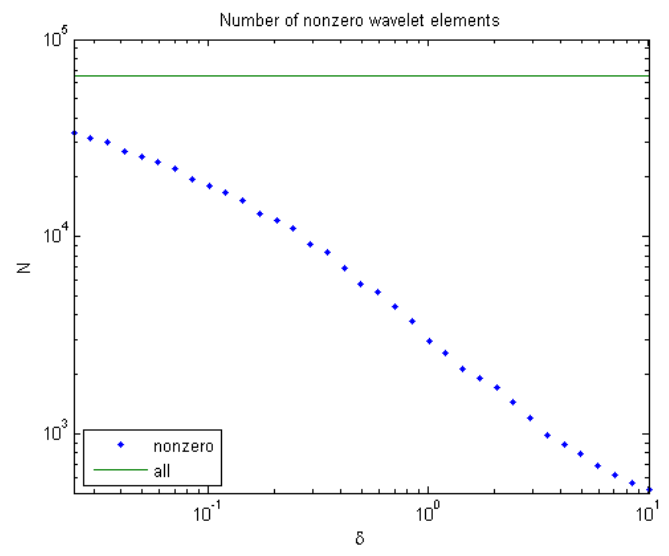

Figure 4. Dashed: Number of nonzero wavelet coefficients in the reconstruction $x(\delta)$ vs. (absolute) noise level. Solid: Full number of coefficients of true solution $x^{\dagger}$.

Finally, as we are using $\ell_{1}$-type regularization, one can also examine the sparsity of the obtained solutions, i.e., the number of non-zero wavelet coefficients. Figure 4 shows how the sparsity develops with respect to decreasing noise levels. The sparsity-promoting effect of the regularization functional can be seen quite well here. Especially in the high noise level case, the number of the reconstructed wavelet coefficients is much lower than the full number of coefficients, which may yield a significant complexity reduction in comparison to the case where a $\ell_{1}$-type regularization is not utilized. 


\section{Conclusions}

The purpose of the paper was to develop a regularization algorithm which combines minimization techniques for Tikhonov-type functionals with discrepancy-based a-posteriori parameter choice rules. It bases, on the one hand, on computable estimates for the discrepancy of the exact minimizers in terms of the discrepancy and the primal-dual gap evaluated at the iterates of a given minimization algorithm. One the other hand, adaptivity of the regularization parameter as well as stopping is achieved by testing against these estimates. It is worth emphasizing that in this framework, the choice of the minimization technique and of the penalty functional is quite arbitrary as long as they fulfill the set of requirements listed in Section 2. We have seen that the procedure establishes a regularization, again under general assumptions. In particular, for weighted $\ell_{p}$-penalty terms, the regularization may also admit a convergence rate.

Previously, the discrepancy rule has mainly been used either for the parameter choice in Tikhonov minimization or for stopping an iterative method. Therefore, our algorithm can be interpreted as one step in closing the gap between Tikhonov-type regularization and iterative regularization techniques. Moreover, the functional formulation behind this framework allows in particular to estimate the approximation quality of the iterates with respect to the exact Tikhonov minimizers. As a side effect, the procedure yields the guarantee that the discrepancy for the obtained approximate solution as well as the associated exact solutions of the Tikhonov functional are within an adjustable bound. Its regularizing effects are independent from the actual minimization algorithm and, consequently, from the number of performed iterations. We tested the approach with the inversion of the convolution operator within the $\ell_{p^{-}}$ regularization framework and compared two minimization techniques, ISTA and monotone FISTA. As expected, MFISTA outperformed ISTA with respect to the computational effort, whereas the quality of regularization remained the same. However, the first order minimization techniques seem to be quite slow and an application of higher-order methods might be useful to speed up the algorithm.

\section{Acknowledgements}

Kristian Bredies gratefully acknowledges support by the Austrian Science Fund (FWF) under grant SFB F32 (SFB “Mathematical Optimization and Applications in Biomedical Sciences”). Mariya Zhariy acknowledges support by the Radon Institute for Computational and Applied Mathematics and by the Johannes Kepler University of Linz during her postdoc term.

\section{References}

[1] Daubechies I, Defrise M and Mol C D 2004 An iterative thresholding algorithm for linear inverse problems with a sparsity constraint Comm. Pure Appl. Math 57 1413-1541 
[2] Beck A and Teboulle M 2009 Fast gradient-based algorithms for constrained total variation image denoising and deblurring problems IEEE Transactions on Image Processing 18 2419-2434

[3] Bonesky T 2009 Morozov's discrepancy principle and Tikhonov-type functionals Inverse Problems 25 015015

[4] Anzengruber S W and Ramlau R 2010 Morozov's discrepancy principle for Tikhonov-type functionals with nonlinear operators Inverse Problems 26025001

[5] Defrise M and Mol C D 1987 A note on stopping rules for iterative regularization methods and filtered SVD in: P. C. Sabatier, ed., Inverse Problems: An Interdisciplinary Study 261-268

[6] Ramlau R, Teschke G and Zhariy M 2008 A compressive Landweber iteration for solving ill-posed inverse problems Inverse Problems 24065013

[7] Zhariy M 2009 Adaptive frame based regularization methods for linear ill-posed inverse problems $P h D$ Thesis, University of Bremen

[8] Neubauer A 1988 An a posteriori parameter choice for Tikhonov regularization in the presence of modelling error Appl. Numer. Math. 4 507-519

[9] Ramlau R 1999 A modified Landweber-method for inverse problems Journal for Numerical Functional Analysis and Optimization 20 79-98

[10] Bonesky T, Dahlke S, Maass P and Raasch T 2010 Adaptive wavelet methods and sparsity reconstruction for inverse heat conduction problems Advances in Computational Mathematics 33(4) 385-411 ISSN 10197168

[11] Bonesky T and Maass P 2009 Iterated soft shrinkage with adaptive operator evaluations J. Inv. and Ill-posed Problems 17(4) 337-358

[12] Raasch T 2009 Sparse reconstructions for inverse PDE problems Structured Decompositions and Efficient Algorithms (Dagstuhl Seminar Proceedings no 08492) ed Dahlke S, Daubechies I, Elad M, Kutyniok G and Teschke G (Dagstuhl, Germany: Schloss Dagstuhl - Leibniz-Zentrum fuer Informatik, Germany) ISSN 1862-4405

[13] Dahlke S, Fornasier M and Raasch T 2009 Multilevel preconditioning for adaptive sparse optimization Tech. rep. DFG SPP 1324 preprint

[14] Burger M and Osher S 2004 Convergence rates of convex variational regularization Inverse Problems 20 $1411-1422$

[15] Resmerita E 2005 Regularization of ill-posed problems in Banach spaces: Convergence rates Inverse Problems 21 1303-1314

[16] Resmerita E and Scherzer O 2006 Error estimates for non-quadratic regularization and the relation to enhancing Inverse Problems 22 801-814

[17] Hofmann B, Kaltenbacher B, Pöschl C and Scherzer O 2007 A convergence rates result for Tikhonov regularization in Banach spaces with non-smooth operators Inverse Problems 23 987-1010

[18] Hein T 2009 On Tikhonov regularization in Banach spaces - optimal convergence rates Applicable Analyis 88 653-667

[19] Hein T 2009 Tikhonov regularization in Banach spaces - improved convergence rates results Inverse Problems 25035002

[20] Neubauer A 2009 On enhanced convergence rates for Tikhonov regularization of nonlinear ill-posed problems in Banach spaces Inverse Problems 25065009

[21] Neubauer A, Hein T, Hofmann B, Kindermann S and Tautenhahn U 2010 Improved and extended results for enhanced convergence rates of Tikhonov regularization in Banach spaces Applicable Analysis 89 17291743

[22] Tautenhahn U 1996 Error estimates for regularization methods in Hilbert scales SIAM J. Numer. Anal. 33 $2120-2130$

[23] Ekeland I and Temam R 1976 Convex Analysis and Variational Problems (Amsterdam: North-Holland)

[24] Showalter R E 1997 Monotone Operators in Banach Space and Nonlinear Partial Differential Equations 
(Mathematical Surveys and Monographs vol 49) (American Mathematical Society)

[25] Bredies K and Lorenz D 2008 Iterated hard shrinkage for minimization problems with sparsity constraints SIAM J. Sci. Comput. 30 657-683

[26] Beck A and Teboulle M 2009 A fast iterative shrinkage-thresholding algorithm for linear inverse problems SIAM J. Imaging Sciences 2 183-202

[27] Griesse R and Lorenz D A 2008 A semismooth Newton method for Tikhonov functionals with sparsity constraints Inverse Problems 24035007

[28] Daubechies I, DeVore R, Fornasier M and Güntürk C S 2010 Iteratively reweighted least squares minimization for sparse recovery Communications on Pure and Applied Mathematics 63 1-38 ISSN 10970312

[29] Figueiredo M A T, Nowak R D and Wright S J 2007 Gradient projection for sparse reconstruction: Application to compressed sensing and other inverse problems IEEE Journal of Selected Topics in Signal Processing $1586-597$

[30] Bredies K and Lorenz D A 2008 Linear convergence of iterative soft-thresholding The Journal of Fourier Analysis and Applications 14 813-837

[31] Tikhonov A N, Leonov A S and Yagola A G 1998 Nonlinear Ill-posed Problems vol I (London: Chapman and Hall)

[32] Grasmair M, Haltmeier M and Scherzer O 2008 Sparse regularization with $\ell^{q}$ penalty term Inverse Problems 24 1-13

[33] Engl H W, Hanke M and Neubauer A 1996 Regularization of Inverse Problems (Dordrecht: Kluwer)

[34] Meyer Y 1992 Wavelets and operators Cambridge Studies in Advanced Math. 37

[35] Werner D 1995 Funktionalanalysis (Berlin-Heidelberg: Springer)

[36] Mannix P 2007 A pair of African penguins, Boulders Beach, South Africa http://www.flickr.com/ photos/paulmannix/552264573/ 